Originalveröffentlichung in: Kerstin P. Hofmann, Hermann Kamp, Matthias Wemhoff (Hg.), Die Wikinger und das Fränkische Reich. Identitäten zwischen Konfrontation und Annäherung (MittelalterStudien des Instituts zur Interdisziplinären Erforschung des Mittelalters und seines Nachwirkens, Paderborn 29), Paderborn 2014, S. 21-50

KERSTIN P. HOFMANN

\title{
Akkulturation und die Konstituierung von Identitäten. Einige theoretische Überlegungen anhand des Fallbeispieles der hogbacks
}

\section{Einleitung}

Kolonialismus ohne große Auswirkungen - ist dies möglich? Diese Frage würde vor dem Hintergrund unserer gesellschaftspolitischen Situation heute wohl kaum jemand so stellen. Umso überraschender fällt Dawn Hadleys positive Antwort für die skandinavische Okkupation des mittelalterlichen Englands aus, der von „Invisible Vikings“ spricht. ${ }^{2}$ Eine der wenigen Fundgattungen, die allgemein als Zeugnisse skandinavischer Herrschaft angesprochen werden - hausförmige Steindenkmäler, die unter dem Begriff hogbacks firmieren - wurden von „colonial monuments " ${ }^{\text {"3 }}$ inzwischen zu "monuments denoting conversion “ ${ }^{\text {“4 }}$ und "tools for assimilation".5 Sind diese also ein materieller Beleg für den scheinbar seltenen Fall einer Akkulturation bei Kolonisten?

Die Folgen von Kulturkontakt für Identitäten sind derzeit jedenfalls ein gesellschaftliches Schlüsselthema und werden daher auch in den verschiedensten Wissen-

1 Bei diesem Aufsatz handelt es sich um eine modifizierte Version eines bereits an anderer Stelle veröffentlichten Manuskriptes: Hogbacks. Zeugnisse akkulturierter Migranten?, in: KAISER, Elke/ SCHIER, Wolfram (Hg.): Mobilität und Wissenstransfer in diachroner und interdisziplinärer Perspektive (Topoi. Berlin Studies of the Ancient World 9), Berlin/Boston 2013, S. 173-208. Es ist die Vorabpublikation einiger Überlegungen, die im Zusammenhang mit einer von mir anvisierten Arbeit über Identitäten und Ur- und Frühgeschichte stehen. Es handelt sich also um work in progress, deren aktuellen Stand der Dinge ich hier ausschnitt- und skizzenhaft zur Diskussion stelle, um dann anhand einer Materialgattung eine von vielen möglichen Interpretationen vorzustellen.

Der Aufsatz entstand im Rahmen meiner Tätigkeit als Koordinatorin der Cross Sectional Group V „Space and Collective Identities“ des Exzellenzclusters 264 „Topoi. The Formation and Transformation of Space and Knowledge", dessen Mitgliedern ich für die zahlreichen anregenden Diskussionen im Rahmen von Workshops, Lesezirkeln etc. sehr zu Dank verpflichtet bin. Für diverse Hinweise, die Einsicht in unpublizierte Manuskripte bzw. Korrekturvorschläge danke ich ganz herzlich: Carmen Marcks-Jacobs, Anne Pedersen, Sabine Pinter, Stefan Schreiber, Jörn Staecker, Jürgen Straub und Felix Wiedemann.

2 Hadley, Dawn: Invisible Vikings, in: British Archaeology 64 (2002), online im Internet: http:// www.britarch.ac.uk/ba/ba64/feat2.shtml (letzter Zugriff: 22.03.2012 um 16:00 Uhr).

3 Lang, James T.: The Hogback: A Viking Colonial Monument, in: Anglo Saxon Studies in Archaeology and History 3 (1984), S. 85-176.

4 STOCKer, David A.: Monuments and Merchants: irregularities in the distribution of stone sculptures in Lincolnshire and Yorkshire in the tenth century, in: HadLeY, Dawn M./Richards, Julian D. (Hg.): Cultures in Contact. Scandinavian Settlement in England in the Ninth and Tenth Centuries, Turnhout 2000, S. 179-212, S. 198.

5 Klayman, Melinda: The Anglo-Scandinavian Hogback: A Tool for Assimilation, online im Internet: http://www.klayperson.com/hogbacks/ (letzter Zugriff: 22.03.2012 um 16:30 Uhr). 
schaften verstärkt diskutiert. Dabei wird einhergehend mit Akkulturation oft von der Gefahr des Identitätsverlusts gesprochen. ${ }^{6}$ Man könnte somit Akkulturation und Identitätskonstituierung leichtfertig als nicht zeitgleich vorkommende und somit unvereinbare Phänomene ansehen. Dies würde jedoch zu einer dualistischen und unterkomplexen Betrachtungsweise führen. Der Untertitel des 2010 auf Schloss Gehrden bei Paderborn stattfindenden Workshops hieß „Zur Dialektik von Akkulturation und skandinavischer Identitätenkonstituierung während der Wikingerzeit". Der ihm innewohnenden Aufforderung zur Reflexion soll daher hier nachgegangen werden. Vor dem Hintergrund räumlich verorteter materieller Kultur gilt es, sich mit den Konzepten Akkulturation und Identität näher auseinanderzusetzen. Als Fallbeispiel dienen mir die eben bereits genannten hogbacks, die zwar nicht dem eigentlichen Referenzgebiet des Workshops entstammen, aber anhand derer einige Aspekte von kulturellem Wandel durch Kulturkontakt m. E. besonders gut beleuchtet werden können.

\section{Identität}

Beim Menschen steht Identität als Selbstheit einer Person oder Gruppe/Entität nicht wie in der Mathematik oder Logik für, vollkommene Gleichheit' oder, Übereinstimmung', sondern wird als dynamische und fragile „Einheit ihrer (diachronen und synchronen) Differenzen " bzw. "Synthesis des Heterogenen " ${ }^{\text {"7 }}$ durch sich von anderen distanzierende sowie zu sich selbst und anderen verhaltene Subjekte gebildet. $^{8}$

Die prozessuale Konstruktion von Identitäten basiert demnach auf einem Wechselspiel zwischen Inklusion und Exklusion und geht stets mit dem Aufbau von Alteritäten - ,übersetzbaren'Andersheiten - und der Abgrenzung zur Alienität - ,radikaler Andersheit - einher (Abb. 1). Die dabei erfolgenden Grenzziehungen können sehr unterschiedlich ausfallen. Sie hängen von der jeweiligen Situation und den Wahrnehmungs- und Bewertungsdifferenzen in den Selbst- und Fremdzuschreibungen ab. ' Identität umfasst sowohl "Gleichheit“ als auch „Differenz“, also einerseits „dazugehören so ähnlich wie“ und anderseits „besonders sein im Unterschied zu". ${ }^{10}$

6 Siehe z. B. Berry, John W.: Acculturation as Varieties of Adaptation, in: PAdilla, Amado M. (Hg.): Acculturation. Theory, Models and Some New Findings, Boulder 1980, S. 9-25, S. 13 f.

7 In Anlehnung an Paul Ricœurs Bezeichnung für den Prozess des ,Emplotment' bei Erzählungen; Ricceur, Paul: Zeit und Erzählung, Bd. 1: Zeit und historische Erzählung, München 1988, S. 106.

8 Straub, Jürgen: Identität, in: Konersmann, Ralf (Hg.): Handbuch Kulturphilosophie, Stuttgart 2012, S. 334-339.

9 Vgl. Brather, Sebastian: Ethnische Interpretationen in der frühgeschichtlichen Archäologie. Geschichte, Grundlagen und Alternativen (RGA, Ergänzungsbd. 42), Berlin/New York 2004, S. $97-103$.

10 Byron, Reginald: Identity, in: BARnARD, Allan/Spencer, Jonathan (Hg.): Encyclopedia of Social and Cultural Anthropology, London/New York 1997, S. 292. 


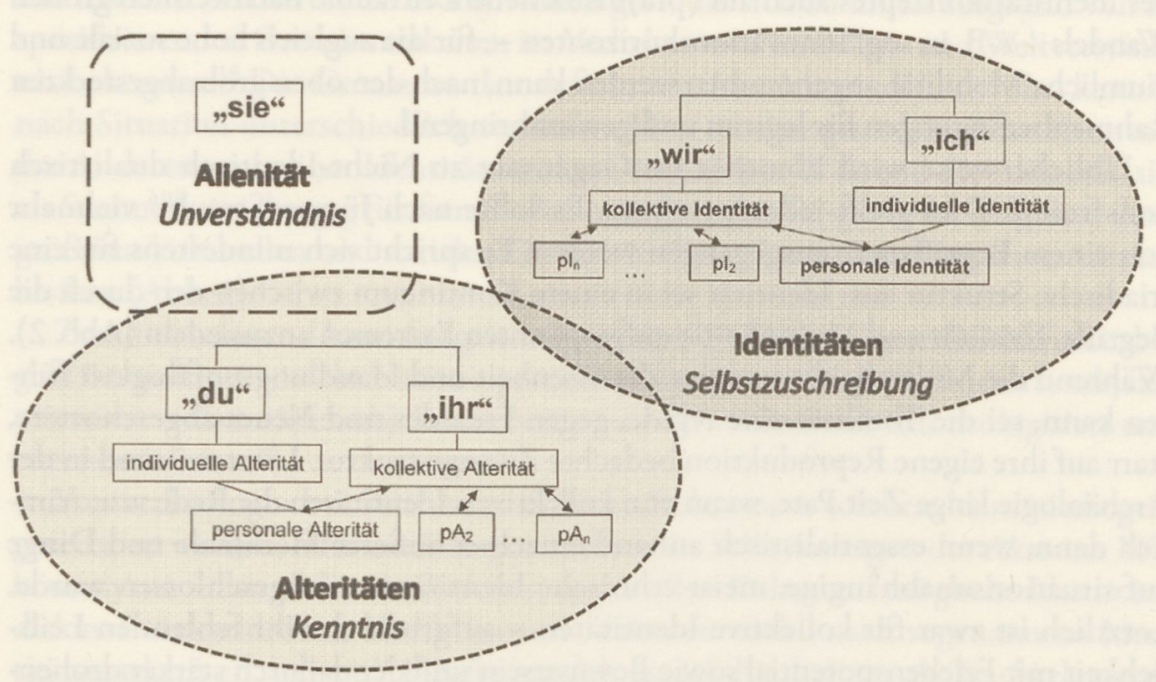

Abb. 1: Identitäten - Alteritäten - Alienität; vgl. AssmanN: Das kulturelle Gedächtnis (Anm. 23), S. 131 .

Die Zugehörigkeiten ergeben sich jedoch nicht allein aus ,bloß Konstruiertem oder aus - mehr oder minder willkürlich - Imaginiertem, sondern basieren auf Erfahrungen, Überzeugungen und Gefühlen der Gruppenmitglieder, ${ }^{11}$ die innerhalb der betreffenden Entität auch ,verdinglicht ' werden können. ${ }^{12} \mathrm{Zu}$ beachten ist jedoch, dass identitäre Gefühle und Gedanken „(lebens-)geschichtlichen Wandel sowie ein in sich vielfältiges Selbst in der Gegenwart mithin eine unabänderlich differentielle, transitorische und heterogene Struktur des Subjekts "13 voraussetzen. Identität ist folglich ein Prozess ständiger Anpassung und Modifikation. Sie spielt allerdings nur dann eine Rolle, wenn sich das Selbst oder das Andere in dynamisch wandelnden Welten differenziert und eine Antwort auf die sich daher stellende Identitäts- bzw. Alteritätsfrage nicht eindeutig ist und somit ein virulentes Problem darstellt. ${ }^{14}$ Deshalb stellt Jürgen Straub ${ }^{15}$ die universale Anwendung des für spätmoderne Gesellschaften der westlichen industrialisierten Welt des 19. Jahrhunderts entwickelten Identitätsbegriffs zu Recht in Frage. Trotz seiner Zweifel an einer sinnvollen Übertragung auf vormoderne Zeiten, halte ich jedoch die Adaption

11 Vgl. Keupp, Heiner/Ahbe, Thomas/Gmür, Wolfgang/HöFer, Renate/Mitzscherlich, Beate/ Kraus, Wolfgang/Straus, Florian: Identitätskonstruktionen. Das Patchwork der Identitäten in der Spätmoderne, Hamburg ${ }^{4} 2008$.

12 Berger, Peter L./Luckmann, Thomas: Die gesellschaftliche Konstruktion der Wirklichkeit. Eine Theorie der Wissenssoziologie, Frankfurt a. M. ${ }^{17} 2000$, S. $94 \mathrm{f}$.

13 Straub: Identität (Anm. 8).

14 Ibid.

15 Straub, Jürgen: Personale und kollektive Identität. Zur Analyse eines theoretischen Begriffs, in: Assmann, Aleida/Friese, Heidrun (Hg.): Identitäten. Erinnerung, Geschichte, Identität, Bd. 3, Frankfurt a. M. 1998, S. 73-104, S. 82 f. 
des Identitätskonzeptes auch auf (prä)historische Zeiträume nachweislich großen Wandels - z. B. in sog. Innovationshorizonten -, für die zugleich hohe soziale und räumliche Mobilität angenommen werden kann, nach den oben grob abgesteckten Rahmenbedingungen für legitim und gewinnbringend.

Üblicherweise wird Identität im Gegensatz zu Nicht-Identisch dualistisch konstruiert. Dies greift jedoch zu kurz. Es sollte nach Jürgen Straub ${ }^{16}$ vielmehr von einem Begriffsnetz ausgegangen werden. Er spricht sich mindestens für eine triadische Struktur aus: Identität sei in einem Kontinuum zwischen den durch die Begriffe Totalität und Multiplizität aufgespannten Extremen anzusiedeln (Abb. 2). Während die Multiplizität zu einer Zerrissenheit und Handlungsunfähigkeit führen kann, sei die Totalität eine regide, gegen Fremdes und Neues abgeschottete, starr auf ihre eigene Reproduktion bedachte Zwangsstruktur. Letztere stand in der Archäologie lange Zeit Pate, wenn von kollektiven Identitäten die Rede war, nämlich dann, wenn essentialistisch anhand einzelner äußerer Merkmale und Dinge auf situationsunabhängige, meist ethnische Identitäten rückgeschlossen wurde. Letztlich ist zwar für kollektive Identitäten - aufgrund der ihr fehlenden Leiblichkeit mit Erlebenspotential sowie Bewusstsein und der dadurch stärker drohenden Fragmentierung - die nähere Positionierung zur Totalität eine immer wieder praktizierte Lösungsstrategie. Diese führt allerdings häufig aufgrund der dadurch bedingten Abkoppelung von Neuerungen sowie der entstehenden Konflikte bei Fremdkontakten zu gravierenden Problemen, die dann meist auch zu einer zeitlichen Begrenzung der Totalität führen.

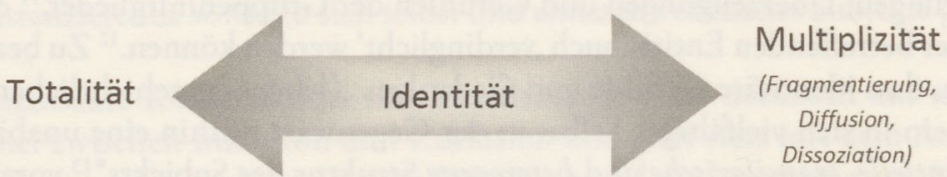

Abb. 2: Die triadische Pragma-Semantik von Identität, Totalität und Multiplizität (vgl. Straub: Identität $[$ Anm. 8]).

Bei jeder kollektiven Identität stellt sich demnach zuallererst immer die Frage nach der Konstitution und Begründung des betreffenden Kollektivs selbst. ${ }^{17}$ Hierfür werden häufig gemeinsame Merkmale, eine für alle ,bindende' und ,verbindliche geschichtliche Kontinuität und praktische Kohärenz angeführt. Ihre Inszenierung und Repräsentation spielen für kollektive Identitäten eine große Rolle. Hierfür sind bleibende ,Lebenselemente - wie Rituale und Routinen, Erzählungen, aber z. B. auch Erbstücke oder Archaika - von besonderer Bedeutung. ${ }^{18}$ Es darf dabei jedoch nicht vergessen werden, dass kollektive Identitäten Konstrukte sind und

16 Straub: Identität (Anm. 8); Ders./Chakkarath, Pradeep: Identität und andere Formen des kulturellen Selbst. Vernunft, Liebe und die Wurzeln der Identität, in: Familiendynamik 35/2 (2010), S. 2-11, S. 6; vgl. ERIKson, Erik H.: Das Problem der Ich-Identität, in: DERS.: Identität und Lebenszyklus. Drei Aufsätze, Frankfurt a.M. 1973, S. 123-224.

17 Straub: Personale und kollektive Identität (Anm. 15), S. 98.

18 Straub: Identität (Anm. 8). 
nur insofern existieren, als es Individuen gibt, die sich zu ihnen aufgrund von zu spezifizierenden Gemeinsamkeiten im Verhalten und im Selbst- und Weltverständnis bekennen. ${ }^{19}$ Die Grenze zu den definierten Alteritäten ,du' und ,ihr ' kann je nach Situation unterschiedlich verlaufen. Hier ist eine grundsätzliche Kenntnis des Anderen und seiner Identitätsstruktur vorhanden. Während die Alienität ,sie als Sammelbezeichnung für fremde Personengruppen fungiert, die aufgrund ihrer größeren kulturellen und sozialen Distanz in ihrer Untergliederung unverstanden bleiben (Abb. 1).

Zusammenfassend kann festgehalten werden: Der hier vorgestellte Identitätsbegriff ist multipel - nicht binär -, prozessual und praxeologisch fundiert, subjektorientiert - aber nicht subjektzentriert -, sondern relational, entontologisiert und entessentialisiert. ${ }^{20}$

Von archäologischer Seite ist es nicht einfach, einem solchen Identitätsbegriff gerecht zu werden, fehlt es doch gewöhnlich an Selbstaussagen der Akteure. Wir können nur anhand der überlieferten materiellen Kultur auf mögliche Identitätsdiskurse rückschließen. Vielfach wurde daher versucht, nach sogenannten Identitätsmarkern zu suchen. ${ }^{21}$ Häufig handelt es sich hierbei um als Trachtelemente angesprochene Kleidungsstücke - insbesondere Fibeln und Gürtel - oder aber um Waffen. ${ }^{22}$ Ein gravierendes Problem ist hierbei jedoch, dass ein etwaiger derartiger Zeichengehalt von Objekten nicht in diese unveränderlich eingeschrieben, sondern er situativ- und kontextgebunden ein Produkt kulturspezifischer Bedeutungszuschreibungen ist. Statt nach statischen Zuständen, gilt es, nach dem materiellen Niederschlag von identitätskonstituierenden Akten und denen von Identitätsdiskursen zu suchen.

In diesem Zusammenhang ist die von Aleida und Jan Assmann ${ }^{23}$ auf Grundlage von Maurice Halbwachs' Konzept der mémoire collective ${ }^{24}$ entwickelte Idee, zwischen ,kommunikativem Gedächtnis' und ,kulturellem Gedächtnis‘ zu unterscheiden, von Interesse. Das kulturelle Gedächtnis würde durch, extra-humane Speicher' wie Monumente, Bilder, Schrift(stücke), aber auch durch Architektur bzw. archäologische Befunde, die durch Repräsentationsakte semiotisch konno-

19 Straub: Personale und kollektive Identität (Anm. 15), S. $102 \mathrm{ff}$.

20 Siehe auch: Gehrke, Hans-Joachim/Hofmann, Kerstin P.: Plenartagungsbericht der Cross Sectional Group V ,Space and Collective Identities', in: Fless, Friederike/Grasshoff, Gerd/MEYer, Michael (Hg.): Berichte der Forschergruppen auf der Topoi-Plenartagung 2010 (eTopoi. Journal for Ancient Studies, Sonderbd. 1 [2011]. http://journal.topoi.org.), S. $5 f$.

21 Vgl. Müller-Scheessel, Nils/Burmeister, Stefan: Einführung: Die Identifizierung sozialer Gruppen. Die Erkenntnismöglichkeiten der Prähistorischen Archäologie auf dem Prüfstand, in: DIES. (Hg.): Soziale Gruppen - kulturelle Grenzen. Die Interpretation sozialer Identitäten in der prähistorischen Archäologie (Tübinger Archäologische Taschenbücher 5), Münster 2006, S. 9-38.

22 Vgl. z.T. äußerst kritisch hierzu: Brather: Ethnische Interpretationen (Anm. 9); BURMEISTER, Stefan: Zum sozialen Gebrauch von Tracht. Aussagemöglichkeiten hinsichtlich des Nachweises von Migrationen, in: Ethnogr-Arch. Zeitschr. 38 (1997), S. 177-203.

23 Vgl. Assmann, Jan: Das kulturelle Gedächtnis. Schrift, Erinnerung und politische Identität in frühen Hochkulturen, München ${ }^{2}$ 1997; AssmanN, Aleida: Erinnerungsräume. Formen und Wandlungen des kulturellen Gedächtnisses, München 1999.

24 Halbwachs, Maurice: Das kollektive Gedächtnis, Frankfurt a. M. ${ }^{4} 1991$. 
tiert sind, gestützt, verdinglicht und könne so längere Zeiträume überbrücken. Die Analyse dieser Zeugnisse für das Selbstverständnis historischer Gemeinschaften bezeichnete Hans-Joachim Gehrke ${ }^{25}$ vor einigen Jahren als Königsweg zur kollektiven Identität. Inzwischen viel rezipiert, ist das allerdings stark nationalistische Konzept der lieux de mémoires, der Erinnerungsorte, des französischen Historikers Pierre Nora. ${ }^{26}$ Nach ihm kristallisiert sich das kollektive Gedächtnis einer sozialen Gruppe an bestimmten ,Orten ' - metaphorisch auch auf Personen, Ereignisse und literarische Werke übertragbar - aus. Diese besitzen eine besonders aufgeladene, symbolische Bedeutung, die für die jeweilige Gruppe identitätsstiftende Funktion hat. Eine Idee, die sicherlich auch gewinnbringend z. B. für Zülpich, Aachen, aber auch Jellinge ${ }^{27}$ ist bzw. schon war, aber auch auf weniger spektakuläre Orte wie Friedhöfe im Allgemeinen angewandt werden kann. Zu beachten ist jedoch, dass Orte und Gegenstände zwar durch ihr Da-Sein zur Reflexion anregen und somit Hilfsmittel für die Aufrechterhaltung eines kulturellen Gedächtnisses sein können, es sich jedoch nicht um Speichermedien im eigentlichen Sinne handelt, denn es bedarf dennoch stets einer kommunikativen Vermittlung. ${ }^{28}$ Auf deren Existenz kann jedoch archäologisch höchstens indirekt rückgeschlossen werden. Indizien hierfür sind u. a. wiederholt an bestimmten Plätzen durchgeführte Rituale oder Raumbezüge zu Vorgängerbauten. Doch schon die Unterscheidung von kontinuierlicher Belegung von Bestattungsplätzen und Wiederaufnahmen bzw. Denkmalbestattungen bereitet mitunter Schwierigkeiten. Ob es sich bei letzteren dann um eine ,invented tradition, ${ }^{69}$ also das Wiederbeleben kultureller Überlieferungen beziehungsweise um einen instrumentalisierten Rückgriff auf die Ressource Vergangenheit, handelt, ist dann meist nur noch mehr oder minder gut spekulierbar. ${ }^{30}$

25 Gehrke, Hans-Joachim: Troia im kulturellen Gedächtnis, in: Zimmermann, Martin (Hg.): Der Traum von Troia. Geschichte und Mythos einer ewigen Stadt, München 2006, S. 211-225, S. 211.

26 NorA, Pierre: Zwischen Geschichte und Gedächtnis. Die Gedächtnisorte, in: DERs. (Hg.): Zwischen Geschichte und Gedächtnis (Kleine Kulturwissenschaftliche Bibliothek 16), Berlin 1990, 11-33.

27 Pollak, Marianne: Vom Erinnerungsort zur Denkmalpflege. Kulturgüter als Medien des kulturellen Gedächtnisses (Studien zu Denkmalschutz und Denkmalpflege 21), Wien 2010, S. 39; vgl. Pedersen, Anne: The Jelling Monuments - ancient royal memorial and modern world heritage site, in: Stoklund, Marie/Lerche Nielsen, Michael/Holmberg, Bente/Fellows-Jensen, Gillian (Hg.): Runes and Their Secrets. Studies in Runology, Copenhagen 2006, S. 283-314.

28 Vgl. DolfF-Bonekämper, Gabi: Memorable moments - chosen cultural affiliations, in: Blatve, Muriel/Gerbel, Christian/Lindenberger, Thomas (Hg.): Clashes in European Memory. The Case of Communist Repression and the Holocaust (Studies in European History and Public Spheres 2), Innsbruck u. a. 2011,S. 143-153.

29 HовSBAwм, Eric: Introduction: Inventing Traditions, in: DERs./RANGER, Terence (Hg.): The Invention of Tradition, Cambridge 1983, S. 1-14.

30 Vgl. Hofmann, Kerstin P.: Kontinuität trotz Diskontinuität? Der Wechsel von der Körper- zur Brandbestattung im Elbe-Weser-Dreieck und die semiotische Bedeutungsebene "Raum“, in: Bérenger, Daniel/Bourgeors, Jean/Talon, Marcel/Wirth, Stefan (Hg.): Gräberlandschaften der Bronzezeit/Paysages funéraires de l'Age du Bronze. Internationales Kolloquium zur Bronzezeit/ Colloque International sur l'Age du Bronze. Herne, 15.-18. Oktober 2008/Herne, 15-18 octobre 2008, Darmstadt 2012, S. 355-373. 


\section{Akkulturation}

Durch Kulturkontakt evozierter Kulturwandel wird insbesondere in den deutschsprachigen Altertumswissenschaften - trotz z. T. heftiger Kritik ${ }^{31}$ - immer noch gern unter dem Oberbegriff, Akkulturation' geführt. ${ }^{32}$ Der in der Ethnologie bzw. cultural anthropology Ende des 19. Jahrhunderts in zahlreichen KulturkontaktStudien allerdings relativ unsystematisch verwendete Terminus ${ }^{33}$ wurde 1932 von Richard Thurnwald als sozio-psychologischer Prozess ${ }^{34}$ und 1935 durch Robert Redfield, Ralph Linton und Melville Herskovits im „Memorandum for the Study of Acculturation " methodologisch fundiert. Die im Memorandum veröffentlichte Definition dient, trotz der seit dem Erscheinen verschiedentlich geäußerten Kritik, ${ }^{35}$ heute immer noch allgemein als Referenzpunkt: „Acculturation comprehends those phenomena which result when groups of individuals having different cultures come into continuous first-hand contact, with subsequent changes in the original patterns of either or both groups " ${ }^{36}$ und dies obwohl sich der Anwendungsbereich

31 Es gibt zahlreiche Kritiker des Akkulturationskonzeptes, z. B. Murphy, Robert F.: Social Change and Acculturation, in: Transactions of the New York Academy of Sciences 26 (1964), S. 845-854; BARTH, Fredrik: On the Study of Social Change, in: American Anthropologist 69 (1967), S. 661669. In der Ethnologie und Soziologie gilt der Akkulturationsansatz als Produkt einer zweifelhaften, strukturfunktionalistischen Vergangenheit zumeist als überholt. Mit ihm sei im Zeitalter des Kolonialismus ein ausbeuterisches soziales System wissenschaftlich unterstützt worden und anstelle seiner simplen Konzeptualisierung von Kulturwandel, bedürfe es vielmehr einer stärker differenzierenden Analytik kulturellen Austausches. Auch wenn ich vielen der vorgebrachten Kritikpunkte zustimme, scheint es mir bis zur Invention eines geeigneteren, politisch nicht so belasteten Begriffes dennoch sinnvoll, gerade in der Archäologie Akkulturation als Oberbegriff für durch Fremdkontakt ausgelösten soziokulturellen Wandel zu verwenden, da viele der neueren Ansätze häufig auf einer in der Archäologie so nicht vorhandenen Quellenbasis fundieren und/oder sie häufig nur bestimmte Aspekte und Relationen näher beleuchten; vgl. ERvin, Alexander M.: A Review of the Acculturation Approach in Anthropology with Special Reference to Recent Change in Native Alaska, in: Journal of Anthropological Research 36 (1980), S. 49-70, S. 49.

32 Z. B. GotTer, Ulrich: ,Akkulturation` als Methodenproblem der historischen Wissenschaften, in: Altekamp, Stefan/Hofter, Matthias René/Krumme, Michael (Hg.): Posthumanistische Klassische Archäologie. Historizität und Wissenschaftlichkeit von Interessen und Methoden, München 2001, S. 255-280; HäGermanN, Dieter/Haubrichs, Wolfgang/JARnut, Jörg (Hg.): Akkulturation. Probleme einer germanisch-romanischen Kultursynthese in Spätantike und frühem Mittelalter (RGA, Ergänzungsbd. 41), Berlin/New York 2004; MEYeR, Marion: Akkulturationsprozesse - Versuch einer Differenzierung, in: DIEs. (Hg.): Neue Zeiten - Neue Sitten. Zur Rezeption und Integration römischen und italischen Kulturgutes in Kleinasien (Wiener Forschungen zur Archäologie 12), Wien 2007, S. 9-18.

33 Vgl. Stagl, Justin: Fremdheit und Akkulturation, in: Neohelicon 23 (1996), S. 61-76, S. 71f. Für einen Überblick zu frühen Verwendungen des Begriffs siehe Herskovits, Melville J.: Acculturation: the Study of Culture Contact, Gloucester Mass. ${ }^{2} 1958$, S. $2-12$.

34 Thurnwald, Richard: The Psychology of Acculturation, in: American Anthropologist 34 (1932), S. 557-569.

35 Siehe Beals, Ralph L. (1953): Acculturation, in: Kroeber, Alfred L. (Hg.): Anthropology today: An Encyclopedic Inventory (Kongress New York 1952), Chicago 1953, S. 621-641, S. 626.

36 Redfield, Robert/Linton, Ralph/Herskovits, Melville J.: Memorandum for the Study of Acculturation, in: American Anthropologist 38/1 (1936), S. 149-152, S. 149. 
des Akkulturationsbegriffes inzwischen wesentlich erweitert hat, z. B. auf, second hand contacts. ${ }^{37}$

Das nach 1935 immer weiter entwickelte Akkulturationskonzept ${ }^{38}$ ist somit letztlich ein Versuch, für die Beschreibung exogen angeregten Kulturwandels ein Analysesystem zur Verfügung zu stellen, welches die Adaption von Entlehnungen thematisiert. Ihm liegt herkömmlich ein holistischer Kulturbegriff zugrunde, der alle Bereiche, auch Politik und Wirtschaft, umfasst, ohne auf die Antithese zwischen Natur und Kultur Bezug zu nehmen. Es stehen sich dabei einzelne, im Wesentlichen hermetisch gedachte Entitäten gegenüber. Dies entspricht jedoch nicht mehr unserem heutigen Verständnis von Gesellschaften, deren dynamische innere Gliederung es stets auch zu berücksichtigen gilt. ${ }^{39}$ Es bedarf also eines Kulturkonzeptes, das ermöglicht, akteursorientiert Wandel durch Kulturkontakt unter Berücksichtigung intrakultureller Heterogenität zu analysieren. Diesen Anforderungen entspricht derzeit m. E. am ehesten der Ansatz von Andreas Wimmer, welcher Kultur als Kompromiss wie folgt definiert: „[... ] so wäre Kultur als ein offener und instabiler Prozeß des Aushandelns von Bedeutungen zu definieren, der kognitiv kompetente Akteure in unterschiedlichen Interessenlagen zueinander in Beziehung setzt und bei einer Kompromissbildung zur sozialen Abschließung und entsprechenden kulturellen Grenzmarkierung führt. ${ }^{\text {“0 }}{ }^{0}$ Die in Kontakt tretenden Kulturen sind demnach nicht Totalitäten, sondern im zuvor beschriebenen komplexen, prozesshaften Verständnis kollektive Identitäten. Durch die damit einhergehende Relationalisierung und Dynamisierung kommt es allerdings mitunter zu Auflösungserscheinungen der in der Definition binär bestimmten Analyseeinheiten, sprich, es fällt schwer bzw. ist unmöglich, z. B. Angel-Sachsen oder Franken und Skandinavier so strikt zu trennen. Dies gilt insbesondere für die langen Zeiträume, die gewöhnlich in der Archäologie untersucht werden.

Im Rahmen der sozialpsychologischen Akkulturationsforschung wurde z. B. festgestellt, dass in kulturellen Überschneidungssituationen - neben Geschlecht, Alter, Status etc. als Einflussfaktoren - bei Personen häufig vier Typen der Verhaltensregulation unterschieden werden können (Abb. 3): Der ,Assimilationstyp

37 Rudolph, Wolfgang: „Akkulturation“ und Akkulturationsforschung, in: Sociologus 14 (1965), S. 97-113, S. 101 f.

38 Z.B. The Social Science Research Council Summer Seminar on Acculturation, 1953: Acculturation: An Exploratory Formulation, in: American Anthropologist 56 (1954), S. 873-1000; BerRY: Acculturation (Anm. 6).

39 GotTer: Akkulturation (Anm. 32), S. 269; siehe auch Koкот, Waltraud: Ethnologische Ansätze zur Akkulturationsforschung, in: HrTzL, Konrad (Hg.): Methodische Perspektiven in der Klassischen Archäologie. Akten der Tagung des Deutschen Archäologen-Verbandes am 19. Juni 2004 in Freiburg (Schriften des Deutschen Archäologen-Verbandes 16), Tübingen 2005, S. 17-25, S. 23.

40 Wimmer, Andreas: Kultur. Zur Reformulierung eines sozialanthropologischen Grundbegriffs, in: KZfSS 48 (1996), S. 401-425, S. 413. Eine ausführlichere Auseinandersetzung mit seinem Kulturbegriff ist in diesem Rahmen leider nicht möglich. Für einen Überblick über die aktuelle Diskussion siehe: SutTER, Alex: Randbemerkungen zu einer Reformulierung des Kulturbegriffs, in: Tsantsa. Zeitschrift der Schweizerischen Ethnologischen Gesellschaft 4 (1999), S. 107-110; MoEbiUs, Stephan/ Quadflieg, Dirk (Hg.): Kultur. Theorien der Gegenwart, Wiesbaden 2006; KHAN-SviK, Gabriele: Kultur - ethnologisch betrachtet, in: Paideia 5/1 (2008), S. 1-21. 


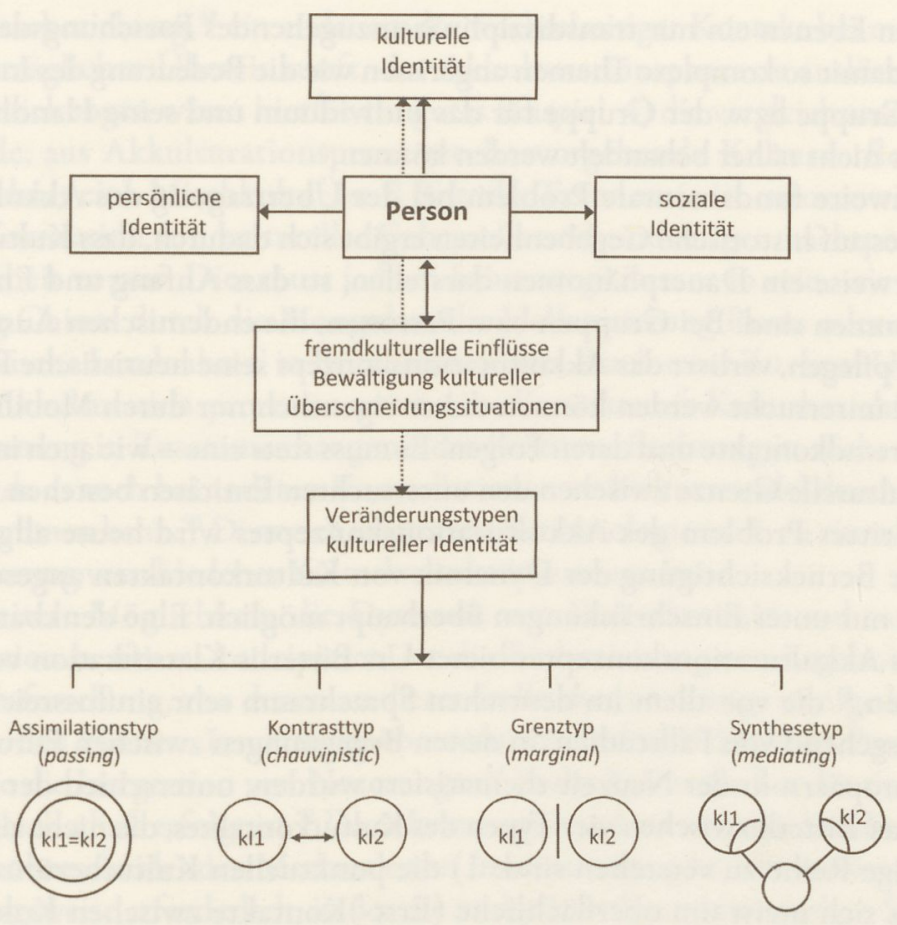

Abb. 3: Veränderungstypen kultureller Identität in kulturellen Uberschneidungssituationen nach Bochner (Grafik von Alexander Thomas leicht modifiziert; online im Internet: www.uni-regensburg.de/ Fakultaeten/phil_Fak_II/Psychologie/Thomas/lehre/Intkultpsychologie/Folien0506/Kapitel_11.ppt - letzter Zugriff: 27.03.2012 um 20:55 Uhr)

lehnt die alte eigene Kultur ab und integriert die Werte, Normen, Verhaltensweisen und Zeichensysteme der neuen Kultur problemlos in sein eigenes Handeln. Der ,Kontrasttyp sieht die eigene Kultur als überlegen an und lehnt die Fremdkultur ab. Für den ,Grenztyp ' haben beide Kulturen bedeutungsvolle Elemente, die für ihn jedoch inkompatibel sind, er schwankt daher ständig zwischen dem eigenen und dem fremden Orientierungssystem. Dem ,Synthesetyp' gelingt es hingegen, Elemente beider Kulturen zu kombinieren, was zu einer multikulturellen Identität führen kann. ${ }^{41}$

Generell stellt sich die Frage, wie einzelne Handlungen, Individuen, personale sowie kollektive Identitäten verschiedener Größenordnungen und ihre jeweiligen Akkulturationsverhalten wechselwirken. Letztlich sind die Interdependenzen der

41 Bochner, Stephen: The social psychology of cross-cultural relations, in: DERs. (Hg.): Cultures in contact: Studies in cross-cultural interactions, Oxford 1982, S. 5-44; siehe auch ThомAs, Alexander: Grundriß der Sozialpsychologie, Bd. 2: Individuum - Gruppe - Gesellschaft, Göttingen 1992, S. 326f.; online im Internet: www.uni-regensburg.de/Fakultaeten/phil_Fak_II/Psychologie/Thomas/ lehre/Intkultpsychologie/Folien0506/Kapitel_11.ppt; zu beachten ist, dass es sich hierbei nicht um eine Persönlichkeitstypologie handelt, sondern nur die gängigsten Einstellungs- und Handlungstendenzen aufzeigt werden sollen, die sich zudem gegenstandsbezogen und situativ verändern können. 
einzelnen Ebenen ein nur transdisziplinär anzugehendes Forschungsdesiderat. Es werden damit so komplexe Themen angerissen wie die Bedeutung des Individuums für die Gruppe bzw. der Gruppe für das Individuum und seine Handlungen, die hier aber nicht näher behandelt werden können.

Das zweite fundamentale Problem bei der Übertragung des Akkulturationskonzeptes auf historische Gegebenheiten ergibt sich dadurch, dass Kulturkontakte üblicherweise ein Dauerphänomen darstellen, so dass Anfang und Ende schwer zu bestimmen sind. Bei Gruppen bzw. Personen, die endemischen Austausch mit anderen pflegen, verliert das Akkulturationskonzept seine heuristische Prägnanz. ${ }^{42}$ Sinnvoll untersucht werden können daher eigentlich nur durch Mobilität entstehende Fremdkontakte und deren Folgen. Es muss stets eine - wie auch immer geartete - kulturelle Grenze zwischen den untersuchten Entitäten bestehen. ${ }^{43}$

Als drittes Problem des Akkulturationskonzeptes wird heute allgemein die fehlende Berücksichtigung der Dynamik von Kulturkontakten angesehen. ${ }^{44} \mathrm{Sie}$ ist m. E. nur unter Einschränkungen überhaupt möglich. Eine denkbare Erweiterung des Akkulturationskonzeptes bietet Urs Bitterlis Klassifikation von Kulturkontakten, ${ }^{45}$ die vor allem im deutschen Sprachraum sehr einflussreich war und ist. ${ }^{46}$ Ausgehend von Fallstudien, in denen Begegnungen zwischen Europäern und Nichteuropäern in der Neuzeit thematisiert wurden, unterschied der Schweizer Historiker Bitterli zwischen vier Typen des Kulturkontaktes, die nicht als genetisch eindeutige Reihe zu verstehen sind: 1) die punktuellen Kulturberührungen, bei denen es sich meist um oberflächliche (Erst-)Kontakte zwischen Kolonisatoren und der indigenen Bevölkerung handelt. 2) den konfliktuellen, mehr oder minder gewaltsamen Kulturzusammenstoß von unterschiedlicher Intensität. Dieser kann von sporadischen Raubzügen bis zur militärischen Eroberung variieren. ${ }^{47}$

42 GotTer: Akkulturation (Anm. 32), S. 268.

43 Gotter, Ulrich: Zwischen Südsee, Paris und Sanssouci. Konzepte von Kulturtransfer und der friderizianische Hof, in: Friedrich300 - Colloquien, Friedrich der Große - Politik und Kulturtransfer im europäischen Kontext. Beiträge des vierten Colloquiums in der Reihe „Friedrich300“ vom 24./25. September 2010. Online im Internet: http://www.perspectivia.net/content/publikationen/ friedrich300-colloquien/friedrich-kulturtransfer/gotter_kulturtransfer (Stand 14.02.2012 um 14:25 Uhr; letzter Zugriff: 25.03 .2012 um 13:45 Uhr), $\$ 31$ und $\$ 32$; vgl. OsterhammeL, Jürgen: Kulturelle Grenzen in der Expansion Europas, in: Saeculum 46 (1995), S. 101-138.

44 GotTer: Akkulturation (Anm. 32), S. 275; Кокот: Ethnologische Ansätze (Anm. 38), S. $23 f$.

45 Bitterli, Urs: Kulturbegegnung und Kulturzusammenstoß in der Sicht des Kolonialhistorikers, in: WendeHorst, Alfred/SCHNeIDER, Jürgen (Hg.): Begegnungsräume von Kulturen. Referate des 4. interdisziplinären Colloquiums des Zentralinstituts (Schriften des Zentralinstituts für Fränkische Landeskunde und allgemeine Regionalforschung an der Universität Erlangen-Nürnberg 21), Neustadt a.d. Aisch 1982, S. 75-88; DERS.: Alte Welt - neue Welt. Formen des europäisch-überseeischen Kulturkontakts vom 15. bis zum 18. Jahrhundert, München 1986, S. 17-54; DERS.: Die,Wilden` und die ,Zivilisierten`. Grundzüge einer Geistes- und Kulturgeschichte der europäisch-überseeischen Begegnung, München ${ }^{2} 1991$, S. 81-179.

46 Siehe z. B. GotTer: Akkulturation (Anm. 32), S. 270ff.; MüNKLER, Marina: Erfahrungen des Fremden. Die Beschreibung Ostasiens in den Augenzeugenberichten des 13. und 14. Jahrhunderts, Berlin 2000, S. 14-20; Osterhammel: Kulturelle Grenzen (Anm. 43), S. $106 f$.

47 Marina Münkler schlug vor, sowohl im Hinblick auf territoriale als auch kulturelle Hegemonieansprüche zwischen peripherem und zentralem Kulturzusammenstoß zu unterscheiden; MüNKLER: 
3) die Kulturbeziehung, ${ }^{48}$ ein andauernder wechselseitiger Kontakt bei mehr oder minder erträglicher Übereinkunft, der Akkulturationsprozesse auslösen kann. Handelsbeziehungen wären hierfür ein gutes Beispiel. 4) die vergleichsweise selten auftretende, aus Akkulturationsprozessen hervorgehende Kulturverflechtung, deren Ergebnis eine Mischkultur ist. ${ }^{49}$ Bitterlis Taxonomie ist eurozentrisch und setzt die Korrelation von kultureller Geberrolle und eindeutiger machtpolitischer Überlegenheit voraus. Dies muss jedoch keineswegs immer so sein, wie z. B. die Eroberung Chinas durch die Mongolen, die Hellenisierung Roms oder eben die ,Skandinavische Landnahme' in Britannien zeigen. Auch seine Zu- bzw. Unterordnung von Akkulturationsprozessen unter seine Kontaktstufe Kulturbeziehung bzw. dessen Nennung in Zusammenhang mit der Kulturverflechtung ist nicht unproblematisch, da es auch bei anderen Konstellationen zu Kulturwandel durch Kulturkontakt kommen kann. ${ }^{50}$ Dennoch trägt seine Terminologie m. E. zu einer besseren Differenzierung verschiedener Kulturkontaktsituationen bei.

Eine weitere Möglichkeit, die Dynamik von Kulturkontakt und somit von Akkulturation besser zu konzipieren, ist die Einbeziehung von Ergebnissen der Innovationsforschung, von denen an dieser Stelle aber nur einige wenige kurz vorgestellt werden können. Innovationsübernahmen sind vielschichtige Prozesse, an deren Ende die Integration von Neuerungen ${ }^{51}$ in das kulturelle Set der sie aufnehmenden Gesellschaft erfolgt ist. ${ }^{52} \mathrm{Da}$ es keine von vornherein feststehenden, für die Übernahme entscheidenden Merkmale von Innovationen gibt, ist eine kontextuelle Betrachtungsweise erforderlich, in der die in der jeweiligen historischen Situation wirksamen Innovationsmerkmale ermittelt werden können. ${ }^{53}$ Dennoch scheint es gewisse strukturelle Gemeinsamkeiten zu geben, die innerhalb der Innovationsforschung herausgearbeitet wurden. ${ }^{54}$ So lässt sich z. B. der für die Akkulturationsforschung besonders interessante Diffusionsprozess einer Innovation in die Stadien

Erfahrungen (Anm. 43), S. 15 Anm. 4.

48 BitTERLI: Alte Welt - Neue Welt (Anm. 45), S. 42-54.

49 Bitterli: Die,Wilden' (Anm. 45), S. 161-173.

50 GoTTER: Akkulturation (Anm. 32), S. 270ff.

51 Heute wird im Allgemeinen zwischen technischen und sozialen Innovationen unterschieden; vgl. BRAUN-THÜRMANN, Holger: Innovation, Bielefeld 2005, S. 16-29; ZAPF, Wolfgang: Über soziale Innovationen, in: Soziale Welt 40 (1989), S. 170-183. Um auch rituellen Wandel zu fassen, habe ich die Einführung des Begriffes der geistigen Innovation vorgeschlagen. Mit ihm seien neue Wege bezeichnet, Ziele zu erreichen, die die Richtung des geistigen/religiösen Wandels verändern und Probleme besser lösen als frühere Praktiken und die es deshalb wert sind, nachgeahmt und institutionalisiert zu werden. Bei Erweiterung des Begriffes der sozialen Innovation könnte die geistige Innovation auch als deren Sonderfall gelten. Der Mehrwert bei der geistigen Innovation ist jedoch nicht aus ökonomischer Sicht, sondern stets nur innerhalb des jeweils spezifischen Glaubens- beziehungsweise Werte- und Normensystems zu verstehen; vgl. HofmanN: Kontinuität (Anm. 30).

52 Burmeister, Stefan: Innovation, ein semiologisches Abenteuer. Das Beispiel der Hallstattzeit in Südwestdeutschland, in: Archäologische Informationen 22 (1999), S. 241-260, S. 241.

53 Vgl. Lemonnier, Pierre: Elements for an Anthropology of Technology (Anthropological Papers. Museum of Anthropology, University of Michigan 88), Ann Arbor 1992.

54 Vgl. Braun-ThürmanN: Innovation (Anm. 51); BläTtel-Mink, Birgit: Kompendium der Innovationsforschung, Wiesbaden 2006; HoF, Hagen/WEGENROTH, Ulrich (Hg.): Innovationsforschung. Ansätze, Methoden, Grenzen und Perspektiven (Innovationsforschung 1), Hamburg 2007. 
der Wahrnehmung, Überzeugung, Entscheidung und Bestätigung aufgliedern (Abb. 4). Die Entscheidungsfindung und die Adaptionsrate, also die benötigte Zeit zur Durchsetzung einer Innovation, werden gleich von einer Reihe von Faktoren beeinflusst. Zum einen spielen die Innovation selbst und die ihr zugeschriebenen Eigenschaften eine Rolle. ${ }^{55}$ Für die Akzeptanz einer Innovation ist es zum Beispiel förderlich, wenn die Neuerung einen relativen Vorteil verspricht und sie mit den Normen, Erfahrungen und Bedürfnissen der potentiellen Adaptoren korrespondiert. Zum anderen ist aber auch die Innovationsbereitschaft der Gemeinschaft von zentraler Bedeutung. ${ }^{56}$ Modellhaft wird hier oft zwischen ,traditionellen und ,modernen ' Gesellschaften unterschieden. ${ }^{57}$ Einschränkend ist jedoch festzuhalten, dass sich moderne Industriegesellschaften zwar im Verhältnis zu Agrargesellschaften sicherlich durch eine höhere Innovationsbereitschaft auszeichnen, dies muss jedoch nicht für alle Situationen und auf alle Lebensbereiche zutreffen. ${ }^{58}$ Ferner muss die jeweilige Situation, in der sich die zu untersuchende Gemeinschaft befindet, berücksichtigt werden. Im Rahmen von Migrationen dürfte allgemein von einer recht hohen Ambiguitätstoleranz $z^{59}$ und Innovationsbereitschaft auszugehen sein, dennoch können sich gerade auch in diesen Situationen in einzelnen Lebensbereichen reaktionäre Verhaltensweisen herausbilden. Für die Entscheidungsfindung und Adaptionsrate der Innovation ist zudem relevant, auf welche Art die Entscheidung getroffen wird: optional, kollektiv, autoritär oder in Form einer Kontingententscheidung. ${ }^{60}$ Die Übernahme einer Innovation hängt außerdem stets auch von den so genannten Agenten, den Befürwortern beziehungsweise Vertretern oder Gegnern einer Innovation, und ihrer sozialen Stellung ab. Letztendlich

55 Fliegel, Frederick C./Kivlin, Joseph E.: Attributes of Innovations as Factors in Diffusion, in: American Journal of Sociology 72 (1966), S. 235-248.

56 BARGATZKY, Thomas: Die Rolle des Fremden beim Kulturwandel (Hamburger Reihe zur Kultur- und Sprachwissenschaft 12), Hamburg 1978, S. 22 f.

57 Rogers, Everett M./Shoemaker, F. Floyd: Communication of Innovations. A Cross-Cultural Approach, New York/London '1971, S. $223 \mathrm{f}$.

58 Vgl. Albrecht, Hartmut: Innovationsprozesse in der Landwirtschaft. Eine kritische Analyse der agrarsoziologischen ,adoption' und ,diffusion'-Forschung in Bezug auf Probleme der landwirtschaftlichen Beratung, Saarbrücken 1969, S. 98ff.; EspínolA, J. C.: La Hipótesis del Tradicionalismo Ideológico - su Exploración Empírica en la Argentina, in: Revista Paraguaya de Sociología 28 (1973), S. 35-58.

59 In der Sozialpsychologie versteht man darunter die Fähigkeit zu Akzeptanz und Umgang mit unklaren Verhaltensweisen und Situationen. Ein Begriff, der nach Lothar Krappmann auch wichtig in der Identitätsbildung ist; vgl. KRAPPMANN, Lothar: Soziologische Dimensionen der Identität, Stuttgart 1975, S. 150.

60 Vgl. Wüstendörfer, Werner: Die Diffusion von Neuerungen. Aspekte einer Adaptionstheorie und deren paradigmatische Prüfung, Dissertation Universität Erlangen 1974, S. 26f. Hier stellt sich die Frage, wie sich bestimmte Entscheidungsprozesse auf Kulturen im Sinne von Wimmer auswirken und ob Kultur als Kompromiss in ähnlicher Weise konzipiert werden kann; vgl. WIMMER: Reformulierung eines sozialanthropologischen Grundbegriffs (Anm. 41). 


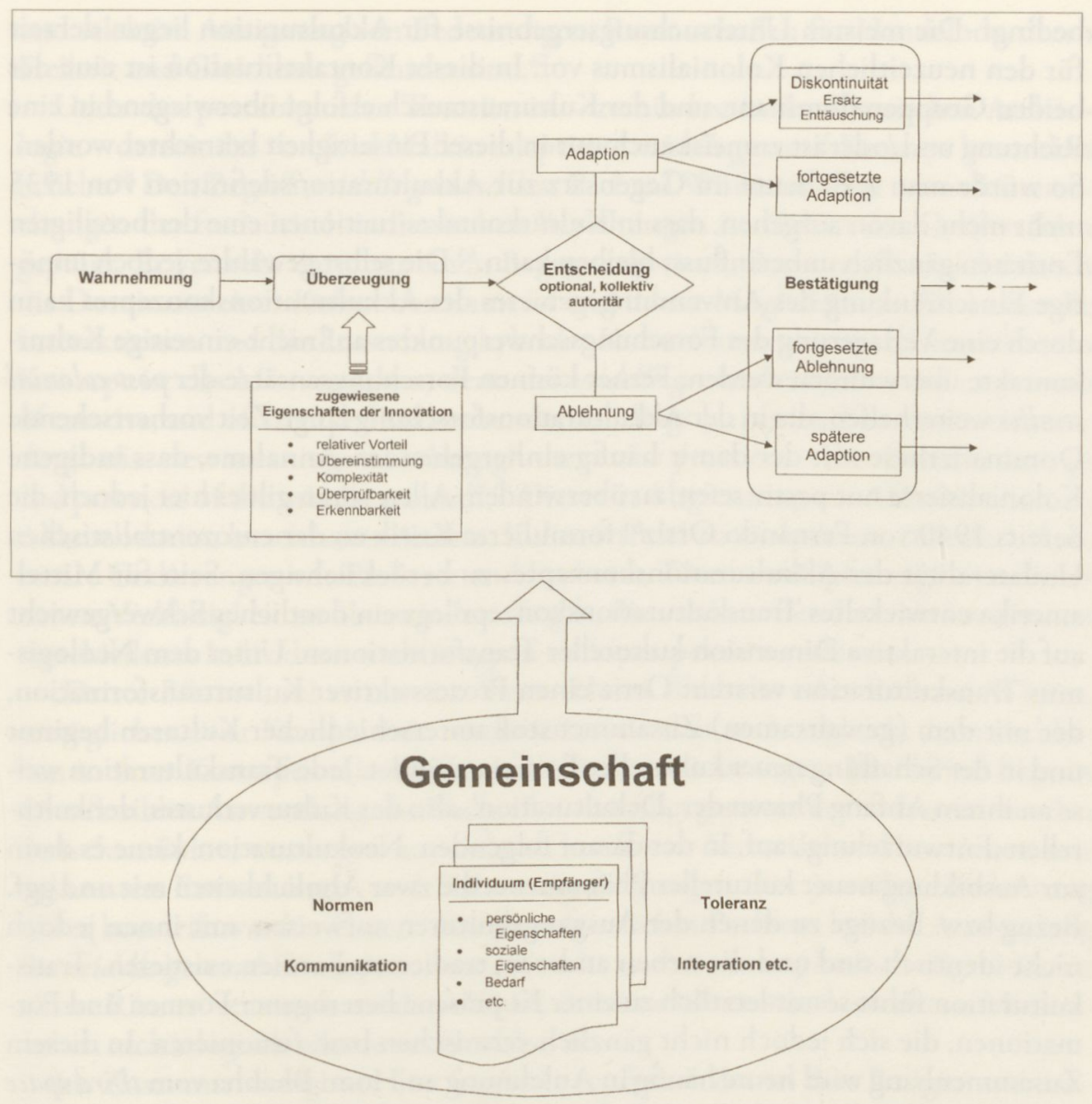

Abb. 4: Entscheidungsprozess für oder gegen die Übernahme einer Innovation nach Rogers/ Shoemaker: Communication of Innovations (Anm. 57), S. 102 mit Abb. [3-1]; Grafik: HofmanN, Kerstin P.: Der rituelle Umgang mit dem Tod. Untersuchungen zu bronze- und früheisenzeitlichen Brandbestattungen im Elbe-Weser-Dreieck (Archäologische Berichte des Landkreises Rotenburg [Wümme] $14=$ Schriftenreihe des Landschaftsverbandes der ehemaligen Herzogtümer Bremen und Verden 32), Oldenburg/Stade 2008, S. 78 mit Abb. 24.

sind auch die verschiedenen Mechanismen kultureller Übermittlung ${ }^{61}$ und die vorhandenen Kommunikationskanäle sowie deren Reichweiten von Bedeutung. ${ }^{62}$

Das vierte und letzte hier behandelte Problem des Akkulturationskonzeptes ist durch dessen Entstehungsbedingungen und die bisherige Forschungsgeschichte

61 Vgl. Krausse, Dirk: Eisenzeitlicher Kulturwandel und Romanisierung im Mosel-Eifel-Raum. Die keltisch-römische Siedlung von Wallendorf und ihr archäologisches Umfeld (Römisch-Germanische Forschungen 63), Mainz am Rhein 2006, S. 51ff. Abb. 17-18.

62 Vgl. WÜstendöRfER: Die Diffusion (Anm. 60), S. $65 \mathrm{ff}$. 
bedingt. Die meisten Untersuchungsergebnisse für Akkulturation liegen derzeit für den neuzeitlichen Kolonialismus vor. In dieser Kontaktsituation ist eine der beiden Gruppen dominant, und der Kulturaustausch erfolgt überwiegend in eine Richtung und/oder ist zumeist auch nur in dieser Einseitigkeit betrachtet worden. So würde man z. B. heute im Gegensatz zur Akkulturationsdefinition von 1935 nicht mehr davon ausgehen, dass in Kulturkontaktsituationen eine der beteiligten Entitäten gänzlich unbeeinflusst bleiben kann. ${ }^{63}$ Die selbst gewählte, jedoch unnötige Einschränkung des Anwendungsgebietes des Akkulturationskonzeptes kann durch eine Verlagerung des Forschungsschwerpunktes auf nicht-einseitige Kulturkontakte überwunden werden. Ferner können Forschungsansätze der post-colonial studies weiterhelfen, die in der Akkulturationsforschung lange Zeit vorherrschende Dominanzthese mit der damit häufig einhergehenden Annahme, dass indigene Kolonialisierte nur passiv seien, zu überwinden. Allen voran gilt es hier jedoch, die bereits 1940 von Fernando Ortiz ${ }^{64}$ formulierte Kritik an der eurozentralistischen Unilateralität des Akkulturationskonzeptes zu berücksichtigen. Sein für Mittelamerika entwickeltes Transkulturationskonzept legt ein deutliches Schwergewicht auf die interaktive Dimension kultureller Transformationen. Unter dem Neologismus Transkulturation versteht Ortiz einen Prozess aktiver Kulturtransformation, der mit dem (gewaltsamen) Zusammenstoß unterschiedlicher Kulturen beginnt und in der Schaffung neuer kultureller Formen mündet. Jede Transkulturation weise an ihrem Anfang Phasen der ,Dekulturation', also des Kulturverlustes, der kulturellen ,Entwurzelung', auf. In der darauf folgenden ,Neokulturation ' käme es dann zur Ausbildung neuer kultureller Phänomene, die zwar Ähnlichkeiten mir und ggf. Bezug bzw. Bezüge zu denen der Ausgangskulturen aufweisen, mit ihnen jedoch nicht identisch sind und die neben anderen, tradierten Formen existieren. Transkulturation führt somit letztlich zu einer Kopräsenz heterogener Formen und Formationen, die sich jedoch nicht gänzlich vermischen bzw. fusionieren. In diesem Zusammenhang wird heute häufig in Anlehnung an Homi Bhabha vom third space gesprochen. ${ }^{65}$ Dabei spielt der im Rahmen der post-colonial studies neukonzeptualisierte Hybriditäts-Begriff eine wichtige Rolle ${ }^{66}$ Er wird auf Situationen kultureller Überschneidung angewandt, in denen teilweise antagonistische Denkinhalte und Logiken aus unterschiedlichen kulturellen, sozialen oder religiösen Lebenswelten zu neuen Handlungs- und Denkmustern zusammengesetzt werden. Nach Homi Bhabha ist Hybridisierung dabei nicht einfach Vermischen, sondern strategische

63 HaLl, Jonathan M.: Hellenicity. Between Ethnicity and Culture, Chicago/London 2002, S. 109.

64 ORTIZ, Fernando: Cuban Counterpoint. Tobacco and Sugar, Durham/London 1995.

65 BнавнA, Homi: The Location of Culture, London 1994; Ders./Rutherford, Jonathan: The Third Space. Interview with Homi Bhabha, in: Rutherford, Jonathan: Identity, Community, Culture, Difference, London 1990, S. 207-221.

66 Vgl. Ackermann, Andreas: Das Eigene und das Fremde: Hybridität, Vielfalt und Kulturtransfer, in: JAEGER, Friedrich/Rüsen, Jörn (Hg.): Handbuch der Kulturwissenschaften, Bd. 3: Themen und Tendenzen, Stuttgart/Weimar 2004, S. 139-154. 
und selektive Aneignung von Bedeutungen, Raum schaffen für Handelnde, deren Freiheit und Gleichheit gefährdet sind. ${ }^{67}$

Unter den postkolonialen Theorien ist - zumindest in der Klassischen Archäologie - derzeit das von Irad Malkin adaptierte middle ground-Konzept besonders beliebt. ${ }^{68}$ Den Begriff, middle ground 'hat Richard White im Zuge seiner Untersuchungen der französisch-indianischen Beziehungen im Gebiet der Großen Seen geprägt. Er versteht darunter einen Begegnungsraum, der - noch - nicht durch die Dominanz einer Kultur bestimmt ist und damit Möglichkeiten für kulturelle Interaktionen und die Entstehung hybrider Kulturformen bietet. Zentral für sein Konzept ist das Missverstehen, denn der middle ground resultiere aus „a process of creative, and often expedient, misunderstandings [...] from these misunderstandings arise new meanings and through them new practices - the shared meanings and practices of the middle ground“ ${ }^{69}$ Dieser in Abgrenzung zur Akkulturation als Akkomodation bezeichnete Ansatz, Kulturwandel zu analysieren, zielt darauf ab, binäre Codierungen wie Eroberer und Eroberte, Täter und Opfer zugunsten einer neuen Vielfältigkeit zu überwinden. Es gilt, die variierenden Interaktionsformen verschiedener Individuen und Gruppen multiperspektiv zu erfassen.

,Dritte Räume', sei es nun im Sinne Homi Bhabahs oder Richard Whites, ermöglichen zwar Komplexität, beschränken sie aber auch zugleich, denn sie bleiben in ihrer Konzeption letztlich auf Dichotomien bezogen. Ihr Vorteil liegt in ihrer flexiblen Anwendung, ihr Nachteil in der Allgegenwart der sie bezeichnenden Zwischenräume, weswegen nur anhand konkreter historischer Situationen mit bekannten Entstehungs- und Rahmenbedingungen ihre jeweilige Bedeutung näher beurteilt werden kann. ${ }^{70}$ Ein grundsätzliches Problem bei der Anwendung des middle-ground-Konzeptes, wenn es nicht nur rein metaphorisch allgemein für ,dritte Räume' verwandt werden soll, ist, dass intendiertes und toleriertes Missverstehen archäologisch schwer nachweisbar ist.

Im Zusammenhang mit den hogbacks ist ferner der in den Religionswissenschaften viel diskutierte Terminus des Synkretismus von Interesse, mit dem die Verschmelzung vorher getrennter Elemente oder Systeme zu einem neuen Gebilde - insbesondere im Bereich der Religion - bezeichnet wird. ${ }^{71}$ Seine Erforschung

67 BHABHA/RutherFord: The Third Space (Anm. 65).

68 WhITE, Richard: The Middle Ground. Indians, Empires, and Republics in the Great Lakes Region, 1650-1815, Cambridge 1991. Vgl. Gosden, Chris: Archaeology and Colonialism. Cultural Contact from 5000 BC to the Present, Cambridge 2004; MaLkin, Irad: The Middle Ground: Philoktetes in Italy, in: Kernos 11 (1998), S. 131-141; MALKIN, Irad: A Colonial Middle Ground: Greek, Etruscan, and Local Elites in the Bay of Naples, in: Lyons, Claire L./PApadopoulos, John K. (Hg.): The Archaeology of Colonialism, Los Angeles 2002, S. 151-181.

69 White: Middle Ground (Anm. 68), S. X.

70 Kienig, Christian: Das wilde Subjekt. Kleine Poetik der Neuen Welt, Göttingen 2006, S. 34.

71 Baliga, Marcolf: Synkretismus und Romanisierung, in: ScHöRnER, Günther (Hg.): Romanisierung - Romanisation. Theoretische Modelle und praktische Fallbeispiele, Oxford 2005, S. 39-44; BERNER, Ulrich: Der Begriff, Synkretismus - ein Instrument historischer Erkenntnis?, in: Saeculum 30 (1979), S. 68-85; Berner, Ulrich: Synkretismus und Inkulturation, in: Siller, Hermann Pius (Hg.): Suchbewegungen. Synkretismus - Kulturelle Identität und kirchliches Bekenntnis, Darmstadt 1991, S. 130-144; CoLPE, Carsten: Die Vereinbarkeit historischer und struktureller Bestimmungen 
zeichnet sich durch eine historische Ausrichtung, den analytischen Blick auf das ,Produkt ' der Vermischung diverser Einflüsse sowie auf die für Identifikationsprozesse so wichtige Berücksichtigung von Definitions- und Legitimationsstrategien ${ }^{72}$ aus.

\section{Fallbeispiel: hogbacks - wikingerzeitliche Steindenkmäler in Großbritannien}

\section{Kurzcharakterisierung}

Unter dem Terminus hogbacks wird in der Archäologie eine recht heterogene Gruppe von wikingerzeitlichen Steindenkmälern Großbritanniens zusammengefasst. ${ }^{73}$ Auf ihre Bedeutung als Quelle für die skandinavische Kolonialisierung der Britischen Inseln machte insbesondere James T. Lang durch seine 1984 erschienene Publikation „The Hogback. A Viking Colonial Monument" aufmerksam. ${ }^{74}$

Hogbacks sind hausförmige Steine von durchschnittlich $150 \mathrm{~cm}$ Länge, $20 \mathrm{~cm}$ Breite und $35 \mathrm{~cm}$ Höhe, wobei jedoch die einzelnen Steine in ihren Maßen sehr

des Synkretismus, in: DieTRICH, Albert (Hg.): Synkretismus im syrisch-persischen Kulturgebiet. Bericht über ein Symposion in Reinhausen bei Göttingen in der Zeit vom 4. bis 8. Oktober 1971 (Abh. der Akademie der Wissenschaften in Göttingen, Philol.-Hist. Klasse 3, Folge 96), Göttingen 1975, S. 15-37. Rudolph, Kurt: Synkretismus - Vom theologischen Scheltwort zum religionswissenschaftlichen Begriff, in: Humanitas Religiosa. FS Harolds Biezais zu seinem 70. Geburtstag dargebracht von Freunden und Kollegen, Stockholm 1979, S. 194-212.

72 WEISsKöPpel, Cordula: 'Hybridität' - die ethnographische Annäherung an ein theoretisches Konzept, in: LoImeier, Roman/Neubert, Dieter/Weissköppel, Cordula (Hg.): Globalisierung im lokalen Kontext. Konzepte und Perspektiven von Handeln in Afrika (Beiträge zur Afrikaforschung 20), Münster/Hamburg/New York/Wien 2005, S. 312-347, S. 331 f.

73 Nach James T. Lang ist ein hogback "a houseshaped recumbent monument of the Viking Age with a definitiv curve to the roof ridge. The roof is usually tegulated and the gables are sometimes embraced by threedimensional animals"; LANG, James T.: Hogback Monuments in Scotland, in: Proceeding of the Society of Antiquaries of Scotland 105 (1972-1974), S. 206-235, S. 206. Ihren Namen erhielten die Steine aufgrund ihrer charakteristischen Form. Zuerst wurden sie aufgrund dieses Merkmals mit dem Rücken von Mastschweinen (back of a hog) verglichen, dann als ,hog backed“ beschrieben; WALKer, Russell: Notes on a Peculiar Class of Recumbent Monuments, in: Proceedings of the Society of Antiquaries of Scotland 19 (1884/1885), S. 406-424, S. 406. Schließlich wurden die beiden Begriffe zu ,hogback'zusammengezogen und als Bezeichnung einer bestimmten Klasse der, recumbent monuments übernommen; LANG: The Hogback (Anm. 3), S. 87.

74 Lang: The Hogback (Anm. 3); siehe auch Abrams, Lesley: The Problem of the Hogback (Draft paper zum Seminar "Myth and cultural memory in the Viking diaspora", 09.-10.01.2007, Leicester). Online im Internet: http://www.nottingham.ac.uk/shared/shared_viking/documents/Abrams.doc (letzter Zugriff: 25.03.2012 13:45 Uhr); BAUs, Annette: Hogback Monuments (Graduate essay Univ. Reading 2008). Online im Internet: http://www.scribd.com/doc/37123893/Hogback-Monuments (letzter Zugriff: 25. 03.2012 13:55 Uhr); KLAYMAN: The Anglo-Scandinavian Hogback (Anm. 5). 
stark variieren können. ${ }^{75}$ Kennzeichnend sind ihre konvexen Seiten. ${ }^{76}$ An den Enden weisen sie mitunter dreidimensionale Tierdarstellungen auf (Abb. 5).

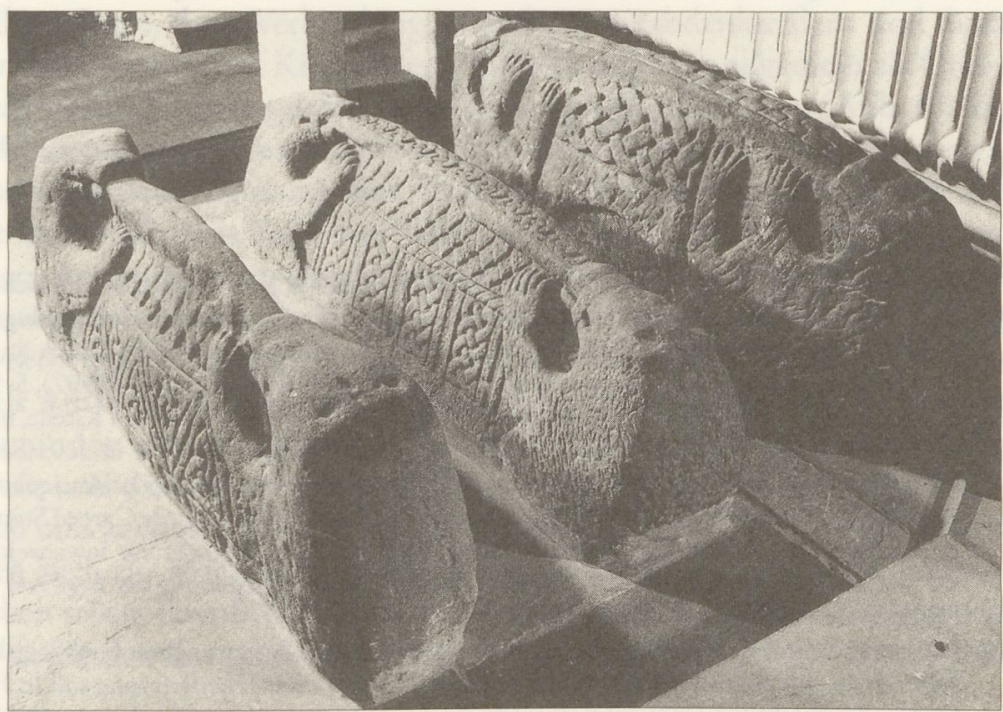

Abb. 5: Hogbacks aus Brompton, North Yorkshire; Richards: Viking-Age England (Anm. 129), S. 124 mit Abb. 81.

Ornamente und Ikonographien sind zahlreich belegt, jedoch keine Inschriften. Von der einst möglicherweise vorhandenen Bemalung ist nichts erhalten. ${ }^{77}$ Überliefert sind 144 hogbacks ${ }^{78}$ meist handelt es sich hierbei aber nur noch um Fragmente. Sie wurden überwiegend im 19. Jahrhundert in alten Kirchen oder auf Kirchhöfen

75 Lang: The Hogback (Anm. 3), S. 87. Der neu gefundene Miniatur-hogback von Bidston mit nur $47,5 \mathrm{~cm}$ Länge, $26,5 \mathrm{~cm}$ Höhe und maximal $15,0 \mathrm{~cm}$ Breite wurde hier nicht berücksichtigt; vgl. BaIley, Richard N./Whalley, Jenny: A Miniature Viking-Age Hogback from Wirral, in: The Antiquaries Journal 86 (2006), S. 345-356. Da vor allem relativ weicher Sand- oder Kalkstein verwendet wurde, ist der überwiegende Anteil der hogbacks stark verwittert, was insbesondere den so genannten Dachbereich betriff; SсHмIDT, Holger: Building Customs in Viking Age Denmark, Herning 1994, S. 138. So können Höhenangaben nur mit Vorbehalt angegeben werden.

76 SснміDт: Building Customs (Anm. 75), S. 138, S. 155.

77 BaIley, Richard N.: Viking Age Sculpture in Northern England, London 1980, S. $26 f$.

78 Die hier angegebene Anzahl basiert auf den in folgenden Publikationen aufgelisteten Fundobjekten: Bailey/Whalley: Miniature Viking-Age Hogback (Anm. 75); Lang, James T.: The Castledermot Hogback, in: Journal of Antiquaries of Ireland 101 (1971), S. 154-158; DeRS.: Hogback Monuments in Scotland (Anm. 73); DeRS.: The Hogback (Anm. 3); NASH-Williams, Victor Earle: The Early Christian Monuments of Wales, Cardiff 1950, S. 98f. Abb. 92. Insgesamt ist die Zuweisung einzelner Steine zu den hogbacks oder den shrine tombs oder recumbent grave slabs umstritten; vgl. ABRAMS: The Problem of the Hogback (Anm. 74), S. 4. Daher kommt es immer wieder zu unterschiedlichen Angaben von Stückzahlen. So zählen Richard Bailey und Jenny Whalley auch nur 119 Exemplare, wobei sie z. B. aber auch keine der schottischen Exemplare berücksichtigen; BAILEY/WHALLEY: Miniature Viking-Age Hogback (Anm. 75), S. 353 Anm. 8. 
gefunden, ${ }^{79}$ die anscheinend auch von überregionaler Bedeutung waren. Keiner der Steine befand sich noch in Originallage ${ }^{80}$ zumeist waren sie sekundär als Baumaterial verwendet worden. ${ }^{81}$

79 So ist z. B. die Kirche von Sockburn schon für das Ende des 8. Jahrhunderts belegt; siehe LANG, James T.: Illustrative Carving of the Viking Period at Sockburn-On-Tees, in: Archaeologia Aeliana Ser. 4/50 (1972), S. 235-248, S. 235; KNowles, William Henry: Sockburn Church, in: Durham and Northumberland Architectural and Archaeological Society Transactions 5 (1907), S. 99-120, S. 99. In Abercorn gab es seit dem 7. Jahrhundert ein Kloster der northumbrischen Kirche, und in Hexham wurde 678 die St. Wilfried's Kirche fertig gestellt; Ross, Thos: Notice of undescribed Hog-backed Monuments at Abercorn and Kirknewton, in: Proceedings of the Society of Antiquaries of Scotland 38 (1904), S. 422-427, S. 426; Collingwood, William Gershom: Early Carved Stones at Hexham, in: Archaeologia Aeliana Ser. 4/1 (1925), S. 65-92, S. 65. Eine Überprüfung, inwieweit obige Aussage auf alle Kirchen zutrifft und diese auch schon zur Zeit der Entstehung der hogbacks als Institution existierten, steht jedoch noch aus.

80 Der einzige Hinweis auf einen möglichen in situ-Fund, eine Bestattung mit Grabbeigabe unter einem hogback, ist mit äußerster Vorsicht zu behandeln. Nach einer Überlieferung soll in Heysham beim Umbau der Kirche um 1800 unter einem hogback eine Lanzenspitze gefunden worden sein. Allerdings gibt es keine Beschreibung der genauen Fundzusammenhänge, zudem ist der Fund in einer weiteren Überlieferung, laut der der hogback um 1820 bei der Anlegung eines Grabes gefunden wurde, nicht erwähnt; siehe EwING, Thor: Understanding the Heysham Hogback: a Tenth-Century Sculpted Stone Monument and its Context, in: Transactions of the Historic Society of Lancashire and Cheshire 152 (2004), S. 1-20, S. 3, S. 16; LAng: The Hogback (Anm. 3), S. 138; Potter, Tim W./Andrews, Richard D.: Excavations and Survey at St Peter's Church, Heysham, Lancashire, 1977-1978, in: Antiquaries Journal 74 (1994), S. 55-134, S. 115; siehe auch: Wenham, Leslie Peter/Hall, Richard A./Briden, C. M./Stocker, David A.: St Mary Bishophill Junior and St Mary Castlegate, in: The Archaeology of York 8/2 (1987), S. 80-83.

81 So wurden sie z. B. sekundär in normannischen Kirchen verbaut; Ross: Notice of undescribed Hogbacked (Anm. 79), S. 425. Eine systematische Untersuchung der Spolierung wäre sicherlich interessant, auch wenn ich im Gegensatz zu Jörn Staecker, der mich freundlicherweise auf diese Perspektive noch einmal aufmerksam machte, die Wiederverwendung der hogbacks nicht notwendigerweise als Indiz für ihren ehemaligen religiösen Kontext betrachten würde. Einige Forschende gehen jedoch davon aus, dass man durch Verbau von Spolien mitunter dem Neubau Autorität und Authenzität verleihen wollte; siehe: BuRström, Mats: Other Generations' Interpretation and Use of the Past: the Case of the Picture Stones on Gotland, in: Current Swedish Archaeology 4 (1996), S. 21-40; Clemens, Lukas: Zum Umgang mit der Antike im hochmittelaltlichen Trier, in: Anton, Hans Hubert/Haverkamp, Alfred (Hg.): 2000 Jahre Trier, Bd. 2: Trier im Mittelalter, Trier 1996, S. $167-$ 202; MEIER, Hans-Rudolf: Vom Siegeszeichen zum Lüftungsschacht. Spolien als Erinnerungsträger in der Architektur, in: DERS./WOHLlEBEN, Marion (Hg.): Bauten und Orte als Träger von Erinnerung. Die Erinnerungsdebatte und die Denkmalpflege, Kongressbericht Zürich 1998 (Veröffentlichungen des Instituts für Denkmalpflege an der ETH Zürich 21), Zürich 2000, S. 87-98, S. 89f.; vgl. allgemein zu Fragen der Spoliierung: POESCHKE, Joachim (Hg.): Antike Spolien in der Architektur des Mittelalters und der Renaissance, München 1996; EsCH, Arnold: Wiederverwendung von Antike im Mittelalter. Die Sicht des Archäologen und die Sicht des Historikers, Berlin/New York 2005; ALtekamP, Stefan/Marcks-Jacobs, Carmen/SeIler, Peter (Hg.): Perspektiven der Spolienforschung, Bd. 1: Spoliierung und Transposition (Topoi. Berlin Studies of Ancient World 15), Berlin/Boston 2013. Für den Einblick in die aktuelle Forschung zum Thema Spoliierung danke ich ganz herzlich Carmen Marcks-Jacobs. 
Aufgrund ihres vergleichsweise hohen Gewichtes ist jedoch von einer Verschleppung der Steine an weiter entfernte Orte abzusehen. ${ }^{82}$ Zudem wurden sie laut geologischen Untersuchungen meist aus lokal anstehendem Gestein hergestellt. ${ }^{83}$

Wegen ihrer Form, ihrer Verbindung zu anderen Grabdenkmälern und ihres postulierten Standortes - auf Kirchhöfen - werden hogbacks allgemein als Grabsteine angesprochen. ${ }^{84}$ Diese gehören zur Quellengruppe Denkmal (Abb. 6). Sie vereinen Merkmale der beiden aufgrund ihrer Intentionalität unterschiedenen Geschichtsquellenarten Überrest und Tradition. Einerseits handelt es sich um Relikte, die unmittelbar als Teil eines umfassenden Totenrituals übrig geblieben sind, andererseits um Quellen, die eigens und absichtlich zur Erinnerung geschaffen worden sind ${ }^{85}$ Grabsteine nehmen damit eine einzigartige Zwischenstellung ein: retrospektiv stellen sie das Gewesene dar; inspektiv geben sie über die Mitund Umwelt Auskunft und prospektiv nehmen sie auf das geglaubte Zukünftige Bezug. ${ }^{86}$ Zumindest zwei grundlegende Funktionen lassen sich unterscheiden: die eschatologische und die kommemorative. ${ }^{87}$ Damit sind häufig Aussagen zur Identität des Toten und auch der Bestattungsgemeinschaft verknüpft. ${ }^{88}$ Mitunter dienen Grabanlagen auch zur Machtlegitimation. ${ }^{89}$ Für die Interpretation eines Grabsteines sind neben seiner räumlichen Verortung seine einzelnen in Wechselbeziehung zueinander stehenden Komponenten - hier technische Ausführung, Form, Orna-

82 Erst in jüngerer Zeit kam es häufiger zum Abtransport von hogbacks. So gelangte der hogback von Barmston nach Lythe, und im Dormitorium der Kathedrale von Durham werden inzwischen unter anderem die hogbacks von Brompton, Arncliff und Easington aufbewahrt. BAILEY: Viking Age Sculpture (Anm. 77), S. 238f.

83 Vgl. Bailey/Whalley: Miniature Viking-Age Hogback (Anm. 75), S. 349.

84 Ritchie, Anna: Viking Scotland, London ${ }^{2} 1994$, S. 97.

85 Vgl. von BRANDT, Ahasver: Werkzeug des Historikers. Eine Einführung in die Historischen Hilfswissenschaften, Stuttgart/Berlin/Köln ${ }^{13} 1992$, S. 48-64; EgGerT, Manfred K. H.: Prähistorische Archäologie. Konzepte und Methoden, Tübingen/Basel ${ }^{4} 2012$, S. 44-49 mit Abb. 4, S. $102 \mathrm{ff}$.

86 Hofmann, Kerstin P.: Gräber und Totenrituale: Zu aktuellen Theorien und Forschungsansätzen, in: Eggert, Manfred K. H./VeIt, Ulrich (Hg.): Theorie in der Archäologie: Zur jüngeren Diskussion in Deutschland (Tübinger Archäologische Taschenbücher 10), Münster/New York/München/Berlin 2013, S. 269-298, S. 273.

87 Vgl. Enninger, Werner/Schwens, Christa: Friedhöfe als kulturelle Texte, in: Zeitschrift für Semiotik 11 (1989), S. 135-181; КосH, Sylvia: Geschichte und Bedeutung des Friedhofs im Abendland, in: ibid., S. 125-133.

88 Vgl. Brather, Sebastian: Bestattungen und Identitäten. Gruppierungen innerhalb frühmittelalterlicher Gesellschaften, in: PoHL, Walter/Mehofer, Mathias (Hg.): Archaeology of Identity - Archäologie der Identität [Kongress Wien 2006] (Österreichische Akademie der Wissenschaften, Phil.-Hist. Klasse Denkschriften 406 = Forschungen zur Geschichte des Mittelalters 17), Wien 2010, S. 25-49; Hofmann, Kerstin P.: Der Identität ihr Grab? Zur Identitätsforschung anhand bronzezeitlicher Bestattungen des Elbe-Weser-Dreiecks, in: Heske, Immo/Horejs, Barbara (Hg.): Bronzezeitliche Identitäten und Objekte. Beiträge aus den Sitzungen der AG Bronzezeit auf der 80. Tagung des Westund Süddeutschen Verbandes für Altertumsforschung in Nürnberg 2010 und dem 7. Deutschen Archäologiekongress in Bremen 2011 (Universitätsforschungen zur prähistorischen Archäologie 221), Bonn 2012, S. 13-25.

89 Vgl. Shanks, Michael/Tilley, Christopher: Ideology, Symbolic Power and Ritual Communication: A Reinterpretation of Neolithic Mortuary Practices, in: Hodder, Ian (Hg.): Symbolic and Structural Archaeology, Cambridge 1982, S. 129-154; DIES.: Social Theory and Archaeology, Cambridge 1987; DIES.: Re-Constructing Archaeology. Theory and Practice, London ${ }^{2} 1992$. 
mentik und Ikonographie - von Bedeutung. Sie sollen im Folgenden auch für die hogbacks näher untersucht werden.

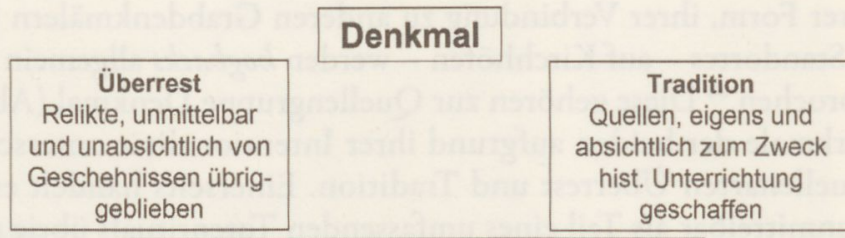

für Lebende und Tote

retrospektiv + inspektiv + prospektiv

eschatologische + kommemorative Funktion

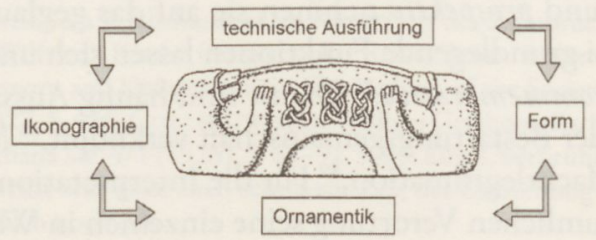

Abb. 6: Grabsteine als historische Quelle (Grafik: Kerstin P. Hofmann).

\section{Verbreitung}

Hogbacks kommen ausschließlich auf den Britischen Inseln vor und hier vor allem in Northumbria (Abb. 7). Nur vereinzelt sind die Steine auch außerhalb von Nordengland und Schottland nachgewiesen. Ihre Form ist dann zumeist durch die regional vertretenen shrine tombs beeinflusst. Skandinavien kennt keine dieser Steindenkmäler, und mit Ausnahme der Gotländischen Bildsteine gibt es auch keine skandinavische Tradition im Bereich der Steinmetzkunst. ${ }^{90}$ Hogbacks sind somit eine Invention des nördlichen Britanniens, und auch ihre Umsetzung bzw. Entwicklung findet anscheinend nur in diesem Gebiet statt. ${ }^{91}$ Die Verbreitung der hogbacks in Britannien erstreckt sich von Küste zu Küste. Die größte Funddichte

90 Abrams: The Problem of the Hogback (Anm. 74), S. 3f. Allerdings gibt es in Skandinavien in späterer Zeit hausförmige Grabmonumente; siehe u. a. ANDERSEN, J. Mørk: En stormandsgrav i Klim, in: Historisk Årbog for Thy (1984), S. 63-76; ANDERSSON, Aron: Gravstenen från Botkyrta och korset från Granhammer, in: Fornvännen 68 (1972), S. 1-12, S. 11; NiLsson, Torben: Sjælens hus, in: Skalk 6 (1994), S. 3-7; STAECKeR, Jörn: Unter einer Hülle Stein. Die wikingerzeitlichen Runensteine und Eskilstunamonumente als historische Quelle, in: DERS. (Hg.): The Reception of Medieval Europe in the Baltic Sea Region. Papers oft he XII ${ }^{\text {th }}$ Visby Symposium held at Gotland University, Visby (Acta Visbyensia 12), Visby 2009, S. 317-378, S. 345-363; TEGNÉR, Göran: Christliche Gräber und Grabdenkmäler, in: RoESDAHL, Else (Hg.): Wikinger - Waränger - Normannen. Skandinavien und Europa 800-1200, 22. Kunstausstellung des Europarats, Mainz 1992, S. 188-189. Für die Zusendung von Literatur(hinweisen) danke ich an dieser Stelle recht herzlich Anne Pedersen und Jörn Staecker.

91 LANG: The Hogback (Anm. 3), S. 95. 
Abb. 7: Verbreitung der hogbacks und kindred monuments auf den Britischen Inseln (Kartierung basiert auf der in Anm. 78 angegebenen Literatur).

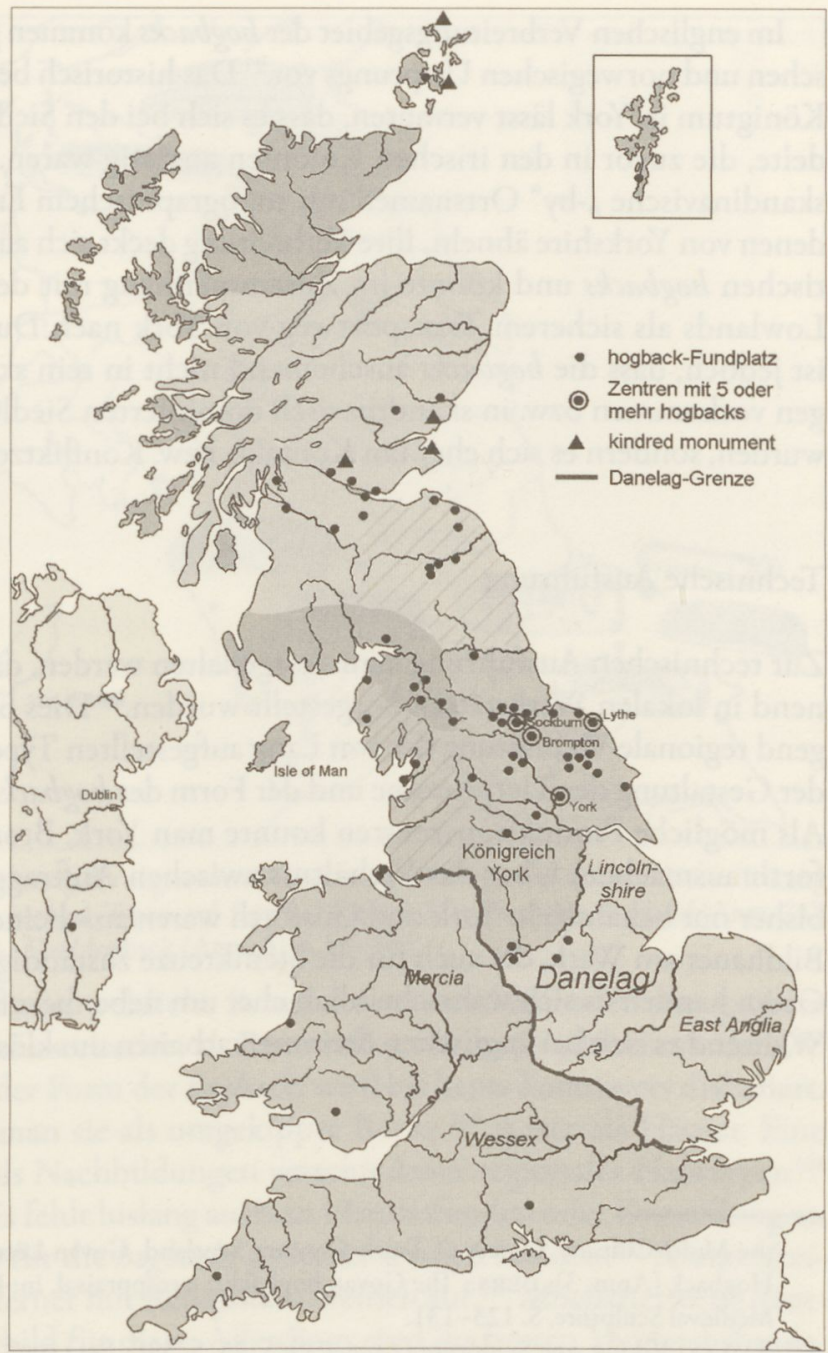

liegt im nördlichen Yorkshire, an Zentren sind hier vor allem Brompton mit elf und das wenige Kilometer entfernt liegende Sockburn mit weiteren neun hogbacks zu erwähnen..$^{92}$ Weitere Konzentrationen befinden sich in Cumbria ${ }^{93}$ mit Gosforth als herausragendem Fundort sowie in Schottland in der Umgebung von Firth of Forth, hier ist vor allem Govan zu nennen. ${ }^{94}$ In einigen Bereichen des Danelags, z. B. Lincolnshire und East Anglia, fehlen sie hingegen ganz.

92 Ibid., S. 87f.

93 BaILey: Viking Age Sculpture (Anm. 77), S. 91.

94 Crawford, Barbara E.: The Norse Background to the Govan Hogbacks, in: Ritchie, Anna (Hg.): Govan and its Early Medieval Sculpture, Stroud 1994, S. 103-112; DIES.: The Govan Hogbacks and 
Im englischen Verbreitungsgebiet der hogbacks kommen gehäuft Ortsnamen gälischen und norwegischen Ursprungs vor. ${ }^{95}$ Das historisch belegte norwegisch-irische Königtum in York lässt vermuten, dass es sich bei den Siedlern um Norweger handelte, die zuvor in den irischen Kolonien ansässig waren. ${ }^{96}$ In Schottland gibt es skandinavische „-by" Ortsnamen mit topographischem Erstglied, die in der Form denen von Yorkshire ähneln. Ihre Verbreitung deckt sich auffällig mit der der schottischen hogbacks und könnte im Zusammenhang mit der Nutzung der Central Lowlands als sicherem Transportweg von York nach Dublin stehen. ${ }^{97}$ Auffällig ist jedoch, dass die hogbacks anscheinend nicht in rein skandinavischen Siedlungen vorkommen bzw. in skandinavisch dominierten Siedlungsarealen angetroffen wurden, sondern es sich eher um Kontakt- bzw. Konfliktzonen handelt. ${ }^{98}$

\section{Technische Ausführung}

Zur technischen Ausführung kann festgehalten werden, dass die hogbacks anscheinend in lokalen Werkstätten hergestellt wurden. ${ }^{99}$ Dies belegt auch die überwiegend regionale Verbreitung der von Lang aufgestellten Typen, die vor allem anhand der Gestaltung der Tierprotome und der Form der hogbacks definiert sind (Abb. 8). Als mögliche Produktionszentren konnte man York, Brompton, Lythe und Gosforth ausmachen. Über das Verhältnis zwischen Auftraggeber und Steinmetz ist bisher nur bekannt: In York und Gosforth waren anscheinend speziell ausgebildete Bildhauer am Werk, die auch für die Steinkreuze zuständig waren. In den kleineren Orten handelt es sich wahrscheinlich eher um nebenberufliche Dorfsteinmetze. ${ }^{100}$ Während es sich bei anglischen Steinmetzarbeiten um klösterliche Kunst handelte,

the Multi-Cultural Society of Tenth-Century Scotland, Govan Lecture, Govan 2005; LANG: The Hogback (Anm. 3); DERS.: The Govan hogbacks: a reappraisal, in: RitchiE: Govan and its Early Medieval Sculpture, S. 123-131.

95 Bailey: Viking Age Sculpture (Anm. 77), S. 35, S. 38f.; Fellows-Jensen, Gillian: The Vikings in England: a review, in: Anglo-Saxon England 4 (1975), S. 181-206, S. 198; DIES.: Place-Names and Settlement in the North Riding of Yorkshire, in: Northern History 14 (1978), S. 19-46, S. 21; DIES.: Vikings in the British Isles: The Place-Name Evidence, in: Acta Archaeologica 71 (2004), S. 135-146; SмYTH, Alfred P.: Scandinavian York and Dublin. The History and Archaeology of two related Viking Kingdoms, Bd. 2, Dublin 1979, S. 280f.; siehe allgemein zur Aussagefähigkeit der onomastischen Quellen in England: ҮокотA, Yumi: What you see is not necessarily what you get: A caveat for Scandinavian place-name evidence, eSharp 2 (2004) online im Internet: http://www.gla. ac.uk/departments/esharp/issues/2/yokota/ (letzter Zugriff 25.03.2012 um 16:45 Uhr).

96 BAILEY: Viking Age Sculpture (Anm. 77), S. 91f.; CrAwFORD: The Govan Hogbacks (Anm. 94), S. 21f.

97 Fellows-Jensen, Gillian: Nordiske spor i det midtskotske Lavland, in: Norna Rapporter 45 (1991), S. 65-83, S. 76-82.

98 Baus: Hogback Monuments (Anm. 74), S. 12ff.

99 BaILEY: Viking Age Sculpture (Anm. 77), S. 242-254.

100 Ibid., S. $254 f$. 


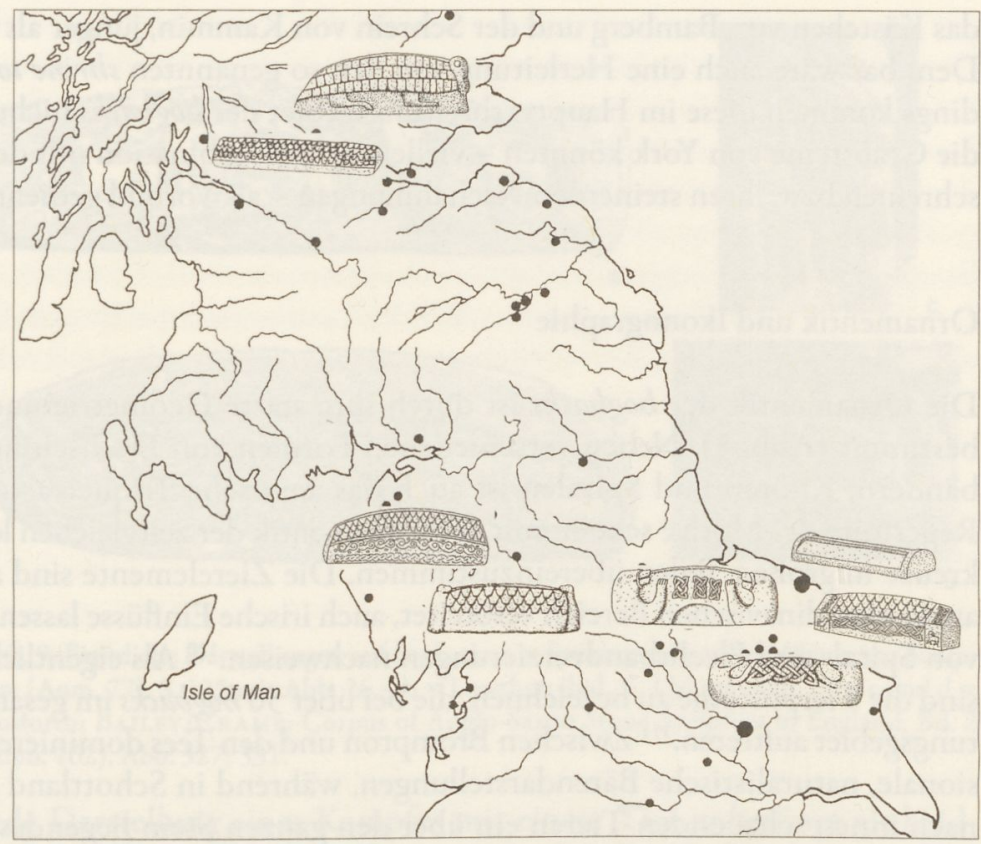

Abb. 8: Kartierung der regionalen Verbreitungsschwerpunkte der von James T. Lang aufgestellten hogback-Typen; vgl. LANG: Hogback Monuments in Scotland (Anm. 73), S. 220; DERS.: The Hogback (Anm. 3), S. 98-102.

spielten in der Wikingerzeit weltliche Schirmherren und Künstler eine größere Rolle, ${ }^{101}$ obwohl die Kirche weiterhin als Patron dieses Kunsthandwerkes auftrat. ${ }^{102}$

Über die Bedeutung der Form der hogbacks wird bis heute kontrovers diskutiert. Anfangs interpretierte man sie als umgekippte Boote, ${ }^{103}$ später als Häuser. Eine Deutung der hogbacks als Nachbildungen verschiedener regionaler Haustypen ${ }^{104}$ ist jedoch abzulehnen. Es fehlt bislang auch an Hausbefunden oder Darstellungen, die als direkte Vorbilder für die hogbacks gedeutet werden können. ${ }^{105}$ Ähnlichkeiten weisen die hogbacks ferner mit Reliquienschreinen auf. ${ }^{106}$ Möglicherweise dienten letztere auch als Vorbild für diese. Allerdings sind die besten Vergleichsfunde,

101 Ibid., S. 82; Graham-Campbell, James (Hg.): Die Wikinger. Weltatlas der alten Kulturen, München 1994, S. 137.

102 Bailey, Richard N./CRAmp, Rosemary: Corpus of Anglo-Saxon Stone Sculpture, Bd. 2: Cumberland, Westmoreland and Lacashire, North-of-the-Sands, Oxford 1988, S. 27.

103 Ross: Notice of undescribed Hog-backed (Anm. 79), S. 423; RowE, G.: Remarks on some ornamental stones found at Brompton, Northallerton, Yorkshire, in: Reports and papers of the architectural and archaeological societies of the counties of Lincoln and Northampton 14 (1877/1878), S. 61-65, S. 62 .

104 Vorgeschlagen von Walton, James: Hogback Tombstones and the Anglo-Danish House, in: Antiquity 28 (1954), S. 68-77.

105 SchmidT: Building Customs (Anm. 75), S. 139.

106 BaIley: Viking Age Sculpture (Anm. 77), S. 94; Lang: Hogback Monuments in Scotland (Anm. 73), S. 206; DERS.: Hogback (Anm. 3), S. 95. 
das Kästchen von Bamberg und der Schrein von Kammin, jünger als die hogbacks. Denkbar wäre auch eine Herleitung von den so genannten shrine tombs, ${ }^{107}$ allerdings kommen diese im Hauptverbreitungsgebiet der hogbacks nicht vor. ${ }^{108}$ Auch die Grabsteine von York könnten - vielleicht in Kombination mit den Reliquienschreinen bzw. ihren steinernen Nachahmungen - als Vorbild gedient haben. ${ }^{109}$

\section{Ornamentik und Ikonographie}

Die Ornamentik der hogbacks ist durch ihre starre Geometrie und Symmetrie bestimmt (Abb. 5). Neben verschiedenen Formen von Dachschindeln, Flechtbändern, Knoten und Spiralen ist auch das anglische Triquetra vertreten. Das Repertoire der Motive scheint mit der Ornamentik der zeitgleichen lokalen Steinkreuze in großen Teilen übereinzustimmen. Die Zierelemente sind allgemein im anglo-skandinavischen Bereich verbreitet, auch irische Einflüsse lassen sich in Form von Spiral- und Flechtbandverzierungen nachweisen. ${ }^{110}$ Als eigentliche Neuerung sind die Tierprotome zu bezeichnen, die bei über 50 hogbacks im gesamten Verbreitungsgebiet auftreten. ${ }^{111}$ Zwischen Brompton und den Tees dominieren dreidimensionale, naturalistische Bärendarstellungen, während in Schottland aus den zwei nach innen schauenden Tieren ein über den ganzen Stein liegendes, nach außen blickendes Tier wurde. ${ }^{112}$

Die Ikonographie der hogbacks ist überwiegend durch Darstellungen von Mythen und Heldenepen bestimmt (Abb. 9). ${ }^{113}$ Exemplarisch seien hierfür zwei hogbacks aus Sockburn und Lowther angeführt. Oft sind bewaffnete Menschen und Kampfszenen dargestellt. Die Detailaufnahme eines hogbacks von Gosforth zeigt das weit verbreitete Motiv ,Kampf Mensch gegen Tier'. Vereinzelt kommt auch christliche Symbolik vor. Auf der Schmalseite eines hogbacks von Gosforth findet sich zum Beispiel ein eingemeißeltes Kruzifix.

Die Ansprache der hogbacks aufgrund der Motivwahl als pagan oder christlich ist problematisch und wird äußerst kontrovers diskutiert. ${ }^{114}$ So muss zum Bei-

107 BaIley: Viking Age Sculpture (Anm. 77), S. 95f.; Collingwood, William Gershom: The Lowther Hogbacks, in: Transactions of the Cumberland and Westmorland Antiquarian and Archaeological Society 7 (1907), S. 152-164, S. 153.

108 Aвrams: The Problem of the Hogback (Anm. 74), S. 3 Anm. 9.

109 Richards, Julian D.: Viking settlement in England, in: BRINK, Stefan (Hg.) in collaboration with Neil Price: The Viking World, London/New York 2008, S. 368-374, S. 370.

110 Siehe Lang: The Hogback (Anm. 3); Bailey/Cramp: Corpus of Anglo-Saxon Stone Sculpture, Bd. 2 (Anm. 102), S. 30; Schmidt: Building Customs (Anm. 75), S. 148.

111 LANG: The Hogback (Anm. 3), S. 106.

112 Bailey: Viking Age Sculpture (Anm. 77), S. 97; Lang: The Hogback (Anm. 3), S. 108.

113 Vgl. BaIley, Richard N.: Scandinavian Myth on Viking-period Stone Sculpture in England, in: Barnes, Geraldine/Ross, Margaret Clunies (Hg.): Old Norse Myths, Literature and Society. Proceedings of the 11th International Saga Conference 2-7 July 2000, University of Sydney, Sydney 2000, S. 15-23.

114 Siehe z. B. Abrams: The Problem of the Hogback (Anm. 74), S. 6f.; Crawford: The Norse Background (Anm. 94); EwING: Understanding the Heysham Hogback (Anm. 80); Hadley, Dawn M.: 

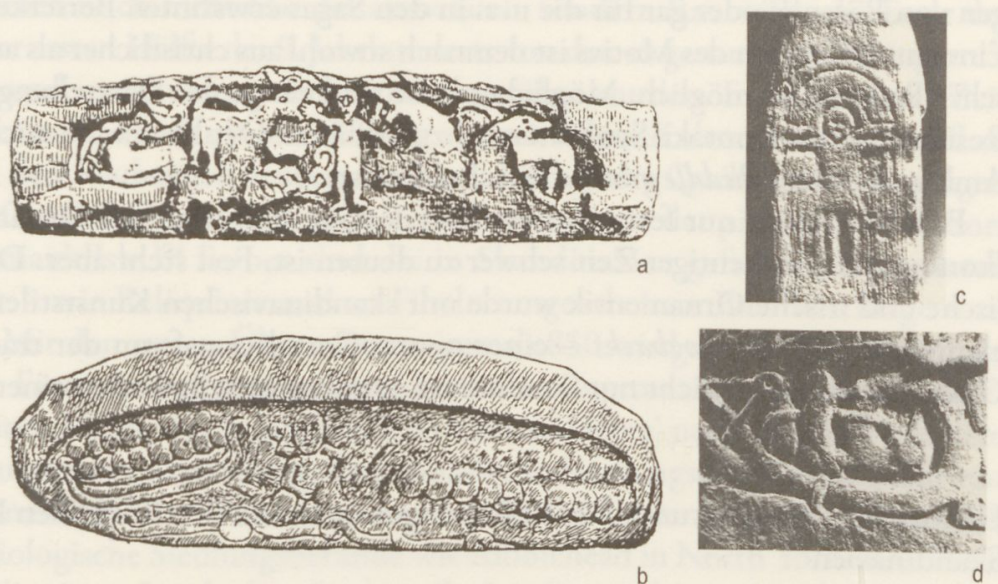

Abb. 9: Figürliche Darstellungen auf hogbacks. a = Sockburn; BAILEY: Viking Age Sculpture (Anm. 77), S. 135 mit Abb. 26 - b = Lowther; ibid., S. 136 mit Abb. $27-\mathrm{c}$ und d = Gosforth; Bailey/Cramp: Corpus of Anglo-Saxon Stone Sculpture in England, Bd. 2 (Anm. 102), Abb. 327; 331.

spiel nicht jede Darstellung eines Kampfes mit einem Tier unbedingt als heidnisch interpretiert werden, denn man findet in der Bibel und in zeitgenössischen christlichen Gedichten eine große Anzahl von Passagen, die durch solche Szenen illustriert werden könnten. Zudem mögen die nordischen Legenden auch genutzt worden sein, um den Fall der heidnischen Götter und den christlichen Sieg über das Böse darzustellen. ${ }^{115}$ Vielseitig deutbar sind auch die Tierprotome. Nach David Stocker sind Bären ein mittelalterliches Symbol der Glaubensbekehrung durch die Kirche. ${ }^{116}$ Sie könnten aber auch in der Tradition der vendelzeitlichen Darstellun-

The Vikings in England. Settlement, Society and Culture, Manchester/New York 2006, S. 222f.; SMYTH: Scandinavian York (Anm. 95), S. 273ff.; STOCKER: Monuments and Merchants (Anm. 4); STONE, Alby: Hogbacks: Christian and pagan imagery on Viking Age monuments, in: 3rd Stone 33 (1999). Online im Internet: http://www.thirdstone.demon.co.uk/download/hogbacks_33.pdf (letzter Zugriff: 05.04.2006), S. 16-20.

115 Siehe z. B. BAILEY: Viking Age Sculpture (Anm. 77), S. 124, S. 140; DERS.: Scandinavian Myth (Anm. 113); Ders./Cramp: Corpus of Anglo-Saxon Stone Sculpture, Bd. 2 (Anm. 102), S. 30, S. 106ff., S. 131; Cramp, Rosemary: Corpus of Anglo-Saxon Stone Sculpture, Bd. 1: County Durham and Northumberland, Oxford/New York 1984, S. 144; Fellows-Jensen, Gillian: The Vikings in England (Anm. 95), S. 204f.; LANG: Illustrative Carving (Anm. 79), S. 247; DERS.: Sigurd and Weland in Preconquest Carving in Northern England, in: Yorkshire Archaeological Journal 48 (1976), S. 83-94, S. 94.

116 STOCKER: Monuments and Merchants (Anm. 4), S. $198 \mathrm{f}$. 
gen von Bären ${ }^{117}$ oder gar für die u. a. in den Sagas erwähnten Berserker stehen. ${ }^{118}$ Eine Interpretation des Motivs ist demnach sowohl aus christlicher als auch heidnischer Perspektive möglich. Möglicherweise sind einige der Darstellungen auch als Resultate eines Kontakt-Synkretismus zu erklären. Denkbar wäre auch, dass ihre Ambiguität dem middle ground zu schulden ist.

Es bleibt somit nur festzuhalten, dass der Symbolgehalt der Ornamentik und Ikonographie in heutiger Zeit schwer zu deuten ist. Fest steht aber: Die alte anglische und irische Ornamentik wurde mit skandinavischen Kunststilen und Bildelementen auf den hogbacks - einer neuen Gestaltungsform der traditionellen Grabmonumente - nicht nur kombiniert, sondern verschmilzt zu einer Einheit. ${ }^{119}$

\section{Historischer Hintergrund: Die Beziehungen zwischen den Britischen Inseln und Skandinavien}

Im Folgenden sollen schlaglichtartig anhand einzelner historischer Daten, archäologischer Funde und onomastischer Quellen die Beziehungen zwischen den Britischen Inseln und Skandinavien betrachtet werden, um so die hogbacks vor ihrem historischen Hintergrund beleuchten zu können. ${ }^{120}$

Den Auftakt für die immer enger werdenden Beziehungen zwischen den Britischen Inseln und Skandinavien bildeten - nach punktuellen Kulturberührun-

$117 \mathrm{Zu}$ nennen wären hier die Matrize von Torslunda, die Darstellungen auf dem Helm Valsgärde 7 und Vendel XI sowie auf dem Taschenbeschlag von Sutton Hoo. Ihre Interpretation ist ebenfalls viel diskutiert; vgl. HaucK, Karl: Altuppsalas Polytheismus exemplarisch erhellt mit Bildzeugnissen des 5.-7. Jahrhunderts (Zur Ikonologie der Goldbrakteaten 53), in: UECKER, Heiko (Hg.): Studien zum Altgermanischen. FS Heinrich Beck (RGA, Ergänzungsbd. 11), Berlin/New York 1994, S. 197-302, S. 214ff.; Greis, Gloria/Polizzotti Geselowitz, Michael N.: Sutton Hoo Art: Two Millennia of History, in: Kendall, Calvin B./Wells Peter S. (Hg.): Voyage to the other world: the legacy of Sutton Hoo, Kongress Minneapolis 1989 (Medieval studies at Minnesota 5), Minneapolis 1992, S. 29-44, S. 36-39.

118 Vgl. HöFler, Otto: Berserker, in: RGA, Bd. 2 ( $\left.{ }^{2} 1976\right)$, S. 298-304; Näsströ M, Britt-Mari: Bärsärkarna. Vikingatidens Elitsoldater, Stockholm 2006.

119 Vgl. Bailey: Viking Age Sculpture (Anm. 77), S. 83f.; Graham-Campbell: Die Wikinger (Anm. 101), S. 137f.

120 Dies geschieht hier zwangsläufig stark simplifizierend, zudem wird bei der Kompilation der Quellen eine im Fokus der Forschung stehende große Erzählung aufgegriffen; vgl. PRIEN, Roland: Archäologie und Migration. Vergleichende Studien zur archäologischen Nachweisbarkeit von Migrationsbewegungen (Universitätsforschungen zur Prähistorischen Archäologie 120), Bonn 2007, S. 188ff.; Wilson, David M.: The Vikings in Britain, in: MüLler-Wille, Michael/SCHNEIDER, Reinhard (Hg.): Ausgewählte Probleme europäischer Landnahmen des Früh- und Hochmittelalters. Methodische Grundlagendiskussion im Grenzbereich zwischen Archäologie und Geschichte, Teil 2, Sigmaringen 1994, S. 81-94. Diese stellt jedoch nur eine von vielen Möglichkeiten dar, die einzelnen überlieferten Indizien zu interpretieren. Für einen detaillierten historischen Überblick siehe: FucHS, Rüdiger: Die Landnahme von Skandinaviern auf den Britischen Inseln aus historischer Sicht, in: Müller-Wille/Schneider: Ausgewählte Probleme europäischer Landnahmen (Anm. 121), S. 95-127; Logan, F. Donald: Die Wikinger in der Geschichte, Stuttgart 1987, S. 159ff; Loyn, Henry R.: The Vikings in Britain, Oxford 1994, S. 30ff.; Roesdahl, Else: Vikingernes verden, Kopenhagen 1993, S. 253ff. 
gen $^{121}$ - vornehmlich nicht Handelsaktivitäten, sondern Raubzüge, die Ende des 8. und in der ersten Hälfte des 9. Jahrhunderts von den so genannten Wikingern ${ }^{122}$ sporadisch und meist unkoordiniert durchgeführt wurden. Es ist in dieser Zeit also weniger von einer Kulturbeziehung als von einem Kulturzusammenstoß zu reden. Von den Raubzügen zeugen nicht nur historische Quellen, wie die Angelsächsische Chronik, ${ }^{123}$ sondern auch Verwahrhorte, wie der piktische Silberhort von St. Ninian's Isle. ${ }^{124}$ Ferner sind die in Skandinavien gefundenen insularen Fundstücke - wie Reliquiare und zu Fibeln umgearbeitete Buchbeschläge - zu nennen. ${ }^{125}$ Mit den ersten Überwinterungen ab 850 wird die Etablierungsphase eingeläutet. Eines der befestigten Lager konnte in Repton ausgegraben werden. ${ }^{126}$ In der zweiten Hälfte des 9. Jahrhunderts ziehen ,Heere' nach England mit dem Ziel der dauerhaften Ansiedlung. Skandinavische Siedlungstätigkeit ist nicht nur durch Ortsnamen ${ }^{127}$ und Personennamen in Rechtstexten belegt, sondern auch durch archäologische Siedlungsbefunde wie Ribblehead in North Yorkshire. ${ }^{128} \mathrm{Als}$ weiteres Indiz einer „Landnahme“ gelten als skandinavisch angesprochene Frauen-

121 Vgl. Wilson, David M.: Wikinger. $\$ 2$. Britische Inseln, in: RGA, Bd. 34 (22007), S. 59-64, S. 59.

122 Zur Problematik des Begriffes,Wikinger siehe Herschend, Frands: Wikinger. $\$ 1$. Historisch, in: RGA, Bd. 34 ( $\left.{ }^{2} 2007\right)$, S. 55-59; KRÜGER, Jana: „Wikinger“ im Mittelalter. Die Rezeption von víkingr $\mathrm{m}$. und víking $\mathrm{f}$. in der altnordischen Literatur (RGA, Ergänzungsbd. 56), Berlin/New York 2008, S. 2ff.; Prien: Archäologie und Migration (Anm. 117), S. 153, S. 188.

123 Whitelock, Dorothy (Hg.): The Anglo-Saxon Chronicle, London 1961; vgl. Fuchs: Landnahme (Anm. 117), S. 101ff.; Logan: Die Wikinger (Anm. 117), S. 38f., S. 160ff.

124 Small, Alan/Thomas, Charles/Wilson, David M.: St. Ninians Isle and its Treasure I-II (Aberdeen University Studies ser. 152), Oxford 1973.

125 Siehe MÜlLER-WILlE, Michael: Das northumbrische Kloster Lindisfarne im Jahre 793 - Die Überfälle von Wikingern auf Klöster der Britischen Inseln und ihre Folgen in der historischen und archäologischen Überlieferung, in: Acta Praehistorica et Archaeologica 34 (2002), S. 225-240; WAMERS, Egon: Insularer Metallschmuck in wikingerzeitlichen Gräbern Nordeuropas. Untersuchungen zur skandinavischen Westexpansion (Offa-Bücher 56), Neumünster 1985.

126 Biddle, Martin/KJøLBYE-Biddle, Birthe: Repton and the Vikings, in: Antiquity 66 (1992), S. 36-51; DIES.: Repton and the ,great heathen army', 873-4, in: Graham-CAMpbell, James/ Hall, Richard/JESCH, Judith/Parsons, David N. (Hg.): Vikings and the Danelaw. Select Papers from the Proceedings of the Thirteenth Viking Congress, Nottingham and York, 21st-30th August 1997, Oxford 2001, S. 45-96.

127 Vgl. Anm. 95. Ein grundsätzliches Problem stellt jedoch die Datierung von skandinavischen Toponymen dar. Zudem variieren die Meinungen über ihre Aussagekraft für Art und Umfang skandinavischer Besiedlung stark; vgl. Үокота: Caveat for Scandinavian Place-Name (Anm. 95).

128 Siehe unter anderem BATEY, Colleen: Aspects of rural settlement in northern Britain, in: BURNELL, Simon/Hooke, Della (Hg.): Landscape and Settlement in Britain AD 400-1066, Exeter 1995; BERESFORD, Guy: Goltho. The Development of an Early Medieval Manor c. 850-1150, London 1987; Coggins, Dennis: Simy Folds: twenty years on, in: Hines, John/Lane, Alan/Redknap, Mark (Hg.): Land, Sea and Home. Proceedings of a Conference on Viking-period Settlement, Cardiff, July 2001 (The Society for Medieval Archaeology monograph ser. 20), Leeds 2004, S. 325-334; HugGins, Peter J.: The Excavation of an eleventh-century Viking hall and fourteenth-century rooms at Waltham Abbey, Essex 1969-1971, in: Medieval Archaeology 20 (1976), S. 75-133; KInG, Alan: Post-Roman upland architecture in the Craven Dales and the dating evidence, in: Hines/LANE/ REDKNAP: Land, Sea and Home, S. 335-344. 
gräber, in denen häufig Schalenfibeln gefunden wurden. ${ }^{129}$ Die gewaltsame Eroberung der Britischen Inseln fand nach der angelsächsischen Rückeroberung Londons Ende des 9. Jahrhunderts ein vorläufiges Ende. Das so genannte Danelag ${ }^{130}$ verlor nach einer ,Friedensperiode' durch den ,kriegerischen Einfluss' der Angelsachsen in der ersten Hälfte des 10. Jahrhunderts rasch an Größe und Einfluss. ${ }^{131} \mathrm{Im}$ Jahre 902 eroberten Iren das norwegische Dublin. Die flüchtenden Skandinavier fanden überwiegend in Nordengland Asyl. ${ }^{132}$ Von den Versuchen, Dublin zurückzuerobern, zeugt vermutlich der um 905 deponierte Cuerdale-Hort. ${ }^{133}$ In den Jahren 919 bis 954 bestand mit Unterbrechung ein mehr oder minder unabhängiges norwegisch-irisches Königtum in York. ${ }^{134}$ Es kommt in dieser Zeit immer mehr zu einer Kulturverflechtung, wobei jedoch gleichzeitig immer wieder auch Kulturzusammenstöße belegt sind. Nach der Vertreibung oder Tötung des letzten norwegischen Königs, Erich Blutaxt, ${ }^{135}$ kehrten einige der Immigranten wahrscheinlich zumindest teilweise wieder nach Skandinavien zurück.

\section{Synthese}

Was sagt dies alles über die hogbacks aus? Bei den hogbacks handelt es sich vermutlich um den materiellen Niederschlag einer Migration. Die mutmaßlichen Grabsteine entstanden wahrscheinlich während der Etablierungsphase der überwiegend wohl aus Irland vertriebenen norwegischen Einwanderer in Yorkshire im zweiten Viertel des 10. Jahrhunderts. ${ }^{136}$ Sie stellen das Resultat eines Akkulturationsprozesses dar. Denn die auf Irland und den Britischen Inseln so verbreiteten Steinskulpturen waren in Skandinavien weitgehend unbekannt. Ferner sind in ihrer Ornamentik skandinavische, anglische und irische Elemente zu einem neuen Kunststil vereint. Die hogbacks wurden anscheinend bei der Konfrontation mit verschiedenen, sich vermutlich politisch, religiös und vielleicht auch ethnisch definierenden

129 PRIEN: Archäologie und Migration (Anm. 117), S. 199; siehe z. B. RichaRdS, Julian D.: Viking-Age England, London 1991, S. $116 f$; Speed, Greg/Rogers, Penelope W.: A burial of a Viking woman at Adwick-le-Street, South Yorkshire, in: Medieval Archaeology 48 (2004), S. 51-90.

130 Siehe Beck, Helmut/Loyn, Henry. R.: Danelag, in: RGA, Bd. 5 ( $\left.{ }^{2} 1984\right)$, S. 227-236.

131 Prien: Archäologie und Migration (Anm. 117), S. $189 \mathrm{f}$.

132 MorRIs, Christopher D.: Northumbria and the Viking Settlement: The Evidence for Land-Holding, in: Archaeologia Aeliana ser. 5/5 (1977), S. 81-103, S. 84 f.

133 Graham-Campbell, James (Hg.): Viking Treasure from the North West. The Cuerdale Hoard and its Context. National Museums and Galleries on Merseyside (Liverpool Museum Occasional Papers 5), Liverpool 1992.

134 Vgl. Hall, Richard A.: Jorvik. Viking age York, York 1980; DERS.: A kingdom too far: York in the early tenth century, in: Higham, Nick J./Hill, David H. (Hg.): Edward the Elder, 899-924, London 2001, S. 188-199.

135 MORRIS: Northumbria (Anm. 129), S. 88f.

136 RichARDS: Viking settlement (Anm. 109), S. 370; zur Datierungsproblematik siehe aber auch Abrams: The Problem of the Hogback (Anm. 74), S. $10 f$. 
Gruppen als Mittel zur Verortung der eigenen Identität genutzt. ${ }^{137}$ Durch ihre Ikonographie wird vor allem das kriegerische Dasein der dargestellten Identität(en) betont. ${ }^{138}$ Vermutlich handelt es sich bei der Personengruppe, die die hogbacks als Repräsentationsmedium nutzte, um Individuen, die nach Stephen Bochner dem ,Synthesetyp ${ }^{\prime 139}$ zugerechnet würden, denn es gelang ihnen, verschiedene Kulturelemente zu kombinieren. Die hogbacks könnten somit Teil der Konstituierung einer - aus der heutigen Vogelperspektive als multikulturell bzw. hybrid zu bezeichnenden - kollektiven Identität gewesen sein.

Eine eindeutige Religionszuweisung anhand der verwendeten Motive kann heute nicht mehr erfolgen. Möglicherweise war diese jedoch auch damals nicht beabsichtigt und bei den hogbacks würde es sich dann um ein charakteristisches Beispiel für materielle Kultur eines , middle grounds ' handeln, bei der Missinterpretationen vorprogrammiert sind. Bei einigen Darstellungen könnte es sich auch um die Resultate eines Kontakt-Synkretimus handeln. Der mutmaßliche Standort - auf alten Friedhöfen regional bedeutender Kirchen - dürfte zumindest auch der Machtlegitimation gedient haben. ${ }^{140}$ Es handelt sich bei den hogbacks demnach um eine entlehnte geistige Innovation, da eine neue Gruppe sich die Platzierung von Grabsteinen inklusive der damit verbundenen Identitätsdiskurse und Machtansprüche aneignete. Form und Bedeutungsinhalte wurden jedoch stark modifiziert. Aufgrund ihrer spezifischen Symbolik hatte diese Innovation nur eine geringe Reichweite. Über ihre jeweilige regional in Form und Ikonographie z. T. sehr unterschiedliche Adaption wurde sicherlich optional von einem kleinen Personenkreis entschieden. Die hogbacks trugen vermutlich schon während ihrer Konzeption und Herstellung zum kommunikativen Gedächtnis bei und mögen auch für die Bestattungsgemeinschaft und ihre Nachkommen als Erinnerungsorte gedient haben. Sie können als Zeugnisse für das Selbstverständnis historischer Personen bzw. Gemeinschaften angesprochen werden. Sicherlich hatten sie zudem das Potential, als Hilfsmittel für die Aufrechterhaltung eines mehrere Generationen überdauernden, kulturellen Gedächtnisses zu dienen. Die Ereignisgeschichte und die baldige Nutzung der hogbacks als Spolien weisen jedoch darauf hin, dass ihnen diese Funktion vermutlich in der Praxis nicht allzu lange zukam.

137 Melinda Klayman spricht die hogbacks als „a tool for Assimilation“ an; vgl. KLayman: The Anglo-Scandinavian Hogback (Anm. 5). Dies würde zumindest Stephen Bochners Verständnis von Assimilation widersprechen, denn der ,Assimilationstyp steht nach ihm der alten eigenen Kultur ablehnend gegenüber und würde demnach auch nicht bewusst Elemente dieser mit der anderer kombinieren; siehe Bochner: Social Psychology (Anm. 41); Thomas: Grundriß Sozialpsychologie (Anm. 41).

138 EwING: Understanding the Heysham Hogback (Anm. 80), S. 20.

139 Siehe Bochner: Social Psychology (Anm. 41); Thomas: Grundriß Sozialpsychologie (Anm. 41).

140 Siehe Stocker: Monuments and Merchants (Anm. 4); Ders./Everson, Paul: Five towns funerals: decoding diversity in Danelaw stone sculpture, in: Graham-CAmpbell/Hall/Jesch/Parsons: Vikings and the Danelaw (Anm. 123), S. 223-243, S. 230ff. Vgl. GondeK, Meggen: Investing in Sculpture. Power in Early-historic Scotland, in: Medieval Archaeology 50 (2006), S. 105-142, S. $107 f$., S. 140. 
Bei dieser weitreichenden Interpretation im Rahmen eines mehr oder minder ereignishistorischen Narratives darf jedoch nicht vergessen werden, dass Quellenstand und -lage ungünstig sind. Vieles beruht ausschließlich auf stilistischen Untersuchungen und der Verknüpfung mit schriftlich Überliefertem. Abgrenzung und Klassifikation der sehr heterogenen Fundgruppe sind problematisch, aber auch die Datierung und Bestimmung der Funktion der hogbacks erweist sich aufgrund fehlender in-situ-Funde als schwierig. Ferner werden so komplexe Fragen, wie die Zuordnung von Funden zu kollektiven Identitäten, die Wechselbeziehung zwischen Glauben und materieller Kultur sowie die Sozialstatus-Zuweisung von Objekten angeschnitten. Trotz dieser Vorbehalte erscheint es mir dennoch sinnvoll, für archäologische Funde und Befunde strukturell plausible Narrative zu entwickeln, um ihre Bedeutung auch für heutige Zeiten aufzuzeigen. Die hier vorgestellte Interpretation ist dabei nur eine von vielen möglichen, die letztlich auf ,unser' Interesse an Wanderungsnarrativen ${ }^{141}$ zurückgeht.

141 Wiedemann, Felix: Völkerwellen und Kulturbringer. Herkunfts- und Wanderungsnarrative in historisch-archäologischen Interpretationen des Vorderen Orients um 1900, in: Ethnogr-Arch. Zeitschr. 51, 1/2 (2010), S. 105-128. Wiedemann, Felix/Hofmann, Kerstin P./Gehrke, HansJoachim (Hg.): Vom Wandern der Völker. Darstellungen und Erzählungen von Migrationen in den Altertumswissenschaften (Topoi. Berlin Studies of Ancient World), Berlin in Vorbereitung. 